\title{
Critères d'irréductibilité pour les représentations des courbes elliptiques
}

\author{
Nicolas Billerey
}

\begin{abstract}
Résumé
Let $E$ be an elliptic curve defined over a number field $K$. We say that a prime number $p$ is exceptional for $(E, K)$ if $E$ admits a $p$-isogeny defined over $K$. The so-called exceptional set of all such prime numbers is finite if and only if $E$ does not have complex multiplication over $K$. In this paper, we prove that the exceptional set is included in the set of prime divisors of an explicit list of integers (depending on $E$ and $K$ ), whose infinitely many of them are non-zero. It provides an efficient algorithm for computing it in the finite case. Other less general but rather useful criteria are given, as well as several numerical examples.
\end{abstract}

\section{Introduction}

Soient $\overline{\mathbf{Q}}$ la clôture algébrique de $\mathbf{Q}$ dans $\mathbf{C}$ et $K$ un corps de nombres contenu dans $\overline{\mathbf{Q}}$. Étant donnés une courbe elliptique $E$ définie sur $K$ et un nombre premier $p$, on note $E[p]$ le groupe des points de $p$-torsion de la courbe $E$. C'est un espace vectoriel de dimension 2 sur le corps $\mathbf{F}_{p}=\mathbf{Z} / p \mathbf{Z}$ muni d'une action du groupe de Galois $\mathrm{G}_{K}=\operatorname{Gal}(\overline{\mathbf{Q}} / K)$. Cela fournit un homomorphisme

$$
\rho_{p}: \mathrm{G}_{K} \longrightarrow \operatorname{Aut}(E[p]) \simeq \mathrm{GL}_{2}\left(\mathbf{F}_{p}\right) .
$$

Serre a démontré ([Ser72]) que si $E$ est sans multiplication complexe sur $\overline{\mathbf{Q}}$, il existe une constante $c(E, K)$ telle que pour tout nombre premier $p>c(E, K)$, la représentation $\rho_{p}$ est surjective. Il a également posé la question (toujours ouverte, y compris pour $K=\mathbf{Q})$ de savoir si $c(E, K)$ peut être choisie indépendamment de $E$ ( Ser79]).

Dans ce travail, on s'intéresse à l'ensemble, noté $\operatorname{Exc}(E / K)$, des nombres premiers $p$ pour lesquels la représentation $\rho_{p}$ ci-dessus est réductible. On dit alors que $p$ est exceptionnel pour le couple $(E, K)$. L'ensemble $\operatorname{Exc}(E / K)$ est généralement fini. Plus précisément, $\operatorname{Exc}(E / K)$ est fini si et seulement $E$ n'a pas de multiplication complexe sur $K$, i.e. $\operatorname{End}_{K}(E)=\mathbf{Z}$ (prop. 2.2). On s'intéresse ici à la question suivante.

Question 1. Le corps $K$ et la courbe $E$ étant donnés, comment déterminer explicitement l'ensemble $\operatorname{Exc}(E / K)$ lorsqu'il est fini?

Lorsque $E$ est sans multiplication complexe, Pellarin ([Pel01]), à la suite de Masser et Wüstholz, a obtenu, comme corollaire de ses travaux, une majoration explicite des nombres premiers exceptionnels. Cependant, en raison des constantes qui y apparaîssent, ce résultat ne se prête malheureusement pas à 
une détermination explicite de l'ensemble des nombres premiers exceptionnels. En utilisant des arguments de théorie du corps de classes, on obtient un critère (théorème 2) portant sur la réduction de $E$ en chaque place finie de $K$ offrant une réponse satisfaisante à la question 1 . De plus, dans le cas où $K$ est de degré impair sur $\mathbf{Q}$, on dispose d'un autre résultat (théorème 1) particulièrement simple à mettre en application.

Ces théorèmes sont illustrés numériquement dans le \$5] où l'on détermine explicitement l'ensemble des nombres premiers exceptionnels de plusieurs courbes elliptiques.

On s'intéresse également dans ce travail à la question suivante.

Question 2. Soient $K$ un corps de nombres et $\mathcal{E}$ un ensemble infini de courbes elliptiques définies sur $K$ tels que pour toute courbe $E$ de l'ensemble $\mathcal{E}, \operatorname{Exc}(E / K)$ soit fini. Peut-on trouver une constante uniforme $\alpha(\mathcal{E}, K)$ telle que pour toute courbe elliptique $E$ appartenant à $\mathcal{E}$, la représentation $\rho_{p}$ soit irréductible dès que $p>\alpha(\mathcal{E}, K)$ ?

Dans le cas où $\mathcal{E}$ est l'ensemble de toutes les courbes elliptiques sans multiplication complexe définies sur $K$, cette question est une étape (importante) vers la résolution de la question uniforme de Serre (voir BP08 pour plus de détails et de nouvelles avancées). Lorsque $K=\mathbf{Q}$ et $\mathcal{E}$ est l'ensemble de toutes les courbes elliptiques définies sur Q, Mazur a montré (Maz78) que tel est le cas avec $\alpha(\mathcal{E}, \mathbf{Q})=163$. Dans le cas où $\mathcal{E}$ est l'ensemble des courbes semi-stables, Kraus a obtenu des résultats uniformes et effectifs pour différentes familles corps de nombres, notamment les corps quadratiques et cubiques ([Kra96, Kra07]). Dans ce travail, on généralise aux corps de nombres plusieurs résultats connus sur $\mathbf{Q}$ (prop. 1.1 et 1.2). On obtient ainsi quelques réponses dans la direction de la question 2 pour des ensembles $\mathcal{E}$ de courbes elliptiques ayant mauvaise réduction additive en une place finie de $K$ et un « défaut de semi-stabilité » particulier. Ces résultats sont particulièrement utiles d'un point de vue numérique et sont illustrés au $\$ 5$.

\section{1 Énoncés des résultats}

\subsection{Une loi utile}

On note $M_{\mathbf{Z}}$ le sous-ensemble de $\mathbf{Z}[X]$ constitué des polynômes unitaires ne s'annulant pas en 0. L'application

$$
\begin{aligned}
& M_{\mathbf{Z}} \times M_{\mathbf{Z}} \longrightarrow \mathbf{Z}[X] \\
&(P, Q) \longmapsto \\
&(P * Q)(X)=\operatorname{Res}_{Z}\left(P(Z), Q(X / Z) Z^{\operatorname{deg} Q}\right)
\end{aligned}
$$

où $\operatorname{Res}_{Z}$ désigne le résultant par rapport à la variable $Z$, définit une loi de monoïde commutatif sur $M_{\mathbf{Z}}$ d'élément neutre $X-1$ (lemme 3.1). De plus, les racines complexes de $P * Q$ sont exactement les produits d'une racine de $P$ par une racine de $Q$, comptées avec multiplicités (loc. cit.). Concrètement, si

$$
P(X)=\prod_{i}\left(X-\alpha_{i}\right) \quad \text { et } \quad Q(X)=\prod_{j}\left(X-\beta_{j}\right),
$$

alors, on a $(P * Q)(X)=\prod_{i, j}\left(X-\alpha_{i} \beta_{j}\right)$. 
Étant donnés $P \in M_{\mathbf{Z}}$ et $k \geq 1$, on convient de noter

$$
P^{* k}=\underbrace{P * \cdots * P}_{k \text { fois }} \quad \text { et } \quad P^{* 0}(X)=X-1 .
$$

Par ailleurs, soient $P \in M_{\mathbf{Z}}$ et $r \geq 1$. Il existe alors un unique polynôme $P^{(r)} \in M_{\mathbf{Z}}$ tel que

$$
P^{(r)}\left(X^{r}\right)=\left(P * \Psi_{r}\right)(X)
$$

où $\Psi_{r}(X)=X^{r}-1$ (lemme 3.2). Les racines complexes de $P^{(r)}$ sont exactement les puissances $r$-ièmes des racines complexes de $P$ comptées avec multiplicités. Autrement dit, si

$$
P(X)=\prod_{i}\left(X-\alpha_{i}\right), \quad \text { alors } \quad P^{(r)}(X)=\prod_{i}\left(X-\alpha_{i}^{r}\right) .
$$

Enfin, l'application $P \mapsto P^{(r)}$ est un morphisme de monoïdes pour la loi $*$.

\section{$1.2 \quad$ Résultats}

On énonce deux résultats dans la direction de la question 1 et deux dans la direction de la question 2 que l'on illustre dans le $\$ 5$ sur des exemples concrets.

On fixe un corps de nombres $K$ contenu dans $\overline{\mathbf{Q}}$ et une courbe elliptique $E$ définie sur $K$. On note $d$ le degré de $K$ sur $\mathbf{Q}, D_{K}$ son discriminant, $\mathcal{O}_{K}$ son anneau d'entiers, $h$ son nombre de classes et $N_{K / \mathbf{Q}}$ la norme de l'extension $K / \mathbf{Q}$.

Soit $\mathfrak{q}$ un idéal premier de $\mathcal{O}_{K}$ en lequel $E$ a bonne réduction. On pose

$$
P_{\mathfrak{q}}(X)=X^{2}-t_{\mathfrak{q}} X+N(\mathfrak{q}) \in \mathbf{Z}[X]
$$

où $N(\mathfrak{q})$ est le cardinal du corps résiduel $\mathcal{O}_{K} / \mathfrak{q}$ et

$$
t_{\mathfrak{q}}=N(\mathfrak{q})+1-A_{\mathfrak{q}},
$$

avec $A_{\mathfrak{q}}$ le nombre de points sur le corps $\mathcal{O}_{K} / \mathfrak{q}$ de la réduction de $E$ en $\mathfrak{q}$.

Soit $\ell$ un nombre premier et

$$
\ell \mathcal{O}_{K}=\prod_{\mathfrak{q} \mid \ell} \mathfrak{q}^{v_{\mathfrak{q}}(\ell)}
$$

la décomposition de $\ell \mathcal{O}_{K}$ en produit d'idéaux premiers de $\mathcal{O}_{K}$. On suppose que $E$ a bonne réduction en chaque idéal premier au-dessus de $\ell$. Par abus de langage, on dit alors que $E$ a bonne réduction en $\ell$. Dans ce cas, on associe à $\ell$ (on rappelle que $E$ et $K$ sont fixés) un polynôme $P_{\ell}^{*}$ à coefficients entiers dont certaines valeurs spéciales vont permettre de déterminer essentiellement l'ensemble $\operatorname{Exc}(E / K)$. Précisément, on pose :

$$
P_{\ell}^{*}=\underset{\mathfrak{q} \mid \ell}{*}\left(P_{\mathfrak{q}}^{\left(12 v_{\mathfrak{q}}(\ell)\right)}\right) \in \mathbf{Z}[X],
$$

où, d'une part le produit $*$, pris au sens de la définition du p1.1 porte sur tous les idéaux premiers de $\mathcal{O}_{K}$ divisant $\ell$ et, d'autre part les exposants $\left(12 v_{\mathfrak{q}}(\ell)\right)$ renvoient à la notation adoptée également au $\S$ précédent. Ce polynôme ne 
dépend que de la famille de triplets d'entiers $\left\{\left(t_{\mathfrak{q}}, v_{\mathfrak{q}}(\ell), f_{\mathfrak{q}}\right)\right\}_{\mathfrak{q} \mid \ell}$ où $N(\mathfrak{q})=\ell^{f_{\mathfrak{q}}}$. Ses racines complexes sont de module $\ell^{6 d}$ (lemme 3.4). On considère alors l'entier (essentiel dans la suite) :

$$
B_{\ell}=\prod_{k=0}^{\left[\frac{d}{2}\right]} P_{\ell}^{*}\left(\ell^{12 k}\right)
$$

où $[d / 2]$ désigne la partie entière de $d / 2$.

Dans la direction de la question 1 de l'Introduction, on montre le critère suivant.

Théorème 1 Soit $p$ un nombre premier exceptionnel pour $(E, K)$. Alors, on est dans l'une des situations suivantes :

1. $p$ divise $6 D_{K}$;

2. il existe un idéal premier $\mathfrak{p}$ de $\mathcal{O}_{K}$ divisant $p$ en lequel $E$ a mauvaise réduction additive avec potentiellement bonne réduction supersingulière.

3. pour tout nombre premier $\ell$ de bonne réduction, le nombre premier $p$ divise l'entier $B_{\ell}($ si $d=1$, on suppose $\ell \neq p)$.

Supposons que $E$ soit donnée par une équation de Weierstrass à coefficients dans l'anneau $\mathcal{O}_{K}$ de discriminant $\Delta_{E}$. On déduit du théorème 1 le corollaire suivant.

Corollaire 1.1 Soit $p$ un nombre premier exceptionnel pour $(E, K)$. Alors, on est dans l'une des situations suivantes:

1. $p$ divise $6 D_{K} N_{K / \mathbf{Q}}\left(\Delta_{E}\right)$;

2. pour tout nombre premier $\ell$ de bonne réduction, le nombre premier $p$ divise l'entier $B_{\ell}($ si $d=1$, on suppose $\ell \neq p)$.

Les racines complexes de $P_{\ell}^{*}$ étant de module $\ell^{6 d}$ (lemme 3.4), on a en particulier :

$$
d \text { impair } \Longrightarrow B_{\ell} \neq 0 \text {. }
$$

On déduit alors du théorème 1 le corollaire suivant.

Corollaire 1.2 (cas du degré impair) On suppose que l'extension $K / \mathbf{Q}$ est de degré d impair. Alors, l'ensemble des nombres premiers exceptionnels pour $E$ est fini. De plus, si $p$ un nombre premier exceptionnel pour $(E, K)$, alors, pour tout nombre premier $\ell$ de bonne réduction, le nombre premier $p$ divise l'entier non nul

$$
6 D_{K} N_{K / \mathbf{Q}}\left(\Delta_{E}\right) B_{\ell} .
$$

Remarque. Plus généralement, on démontre à partir de la proposition 2.2 et de résultats classiques de la théorie de la multiplication complexe que les propriétés suivantes sont équivalentes :

1. le corps $K$ ne contient pas le corps de classes de Hilbert d'un corps quadratique imaginaire;

2. pour toute courbe elliptique $E$ définie sur $K$, l'ensemble $\operatorname{Exc}(E / K)$ est fini. 
La situation est plus compliquée dans le cas des extensions de degré pair. Bien que le critère du théorème 1 ci-dessus s'applique toujours (cf. \$5.3), on n'a plus la garantie, pour une courbe ayant un ensemble exceptionnel fini, qu'il existe un nombre premier $\ell$ de bonne réduction pour lequel l'entier $B_{\ell}$ soit non nul (comme le montre l'exemple 5.8). L'énoncé plus général ci-dessous permet de contourner cette difficulté et constitue notre résultat principal en direction de la question 1.

Théorème 2 Soit $p$ un nombre premier exceptionnel pour $(E, K)$. Alors, on est dans l'une des situations suivantes :

1. $p$ divise $6 D_{K}$;

2. il existe un idéal premier $\mathfrak{p}$ de $\mathcal{O}_{K}$ divisant $p$ en lequel $E$ a mauvaise réduction additive avec potentiellement bonne réduction supersingulière.

3. pour tout idéal premier $\mathfrak{q}$ de bonne réduction, le nombre premier $p$ divise l'entier

$$
R_{\mathfrak{q}}=\prod_{k=0}^{\left[\frac{d}{2}\right]} \operatorname{Res}\left(P_{\mathfrak{q}}^{(12 h)},\left(\mathfrak{m}_{\gamma_{\mathfrak{q}}}^{(12)}\right)^{* k}\right),
$$

où $\mathfrak{q}^{h}=\gamma_{\mathfrak{q}} \mathcal{O}_{K}$ et $\mathfrak{m}_{\gamma_{\mathfrak{q}}}$ est le polynôme minimal de $\gamma_{\mathfrak{q}}$ sur $\mathbf{Q}$ (si $d=1$, on suppose que $\mathfrak{q}$ ne divise pas $p$ ).

De plus, si $E$ est sans multiplication complexe sur $\overline{\mathbf{Q}}$, alors $R_{\mathfrak{q}} \neq 0$ pour une infinité d'idéaux premiers $\mathfrak{q}$.

Dans la direction de la question 2 de l'Introduction, on généralise aux corps de nombres plusieurs résultats connus sur $\mathbf{Q}$. Soit $\mathfrak{q}$ un idéal premier de $\mathcal{O}_{K}$ de caractéristique résiduelle $\ell$. On a

$$
N(\mathfrak{q})=\left|\mathcal{O}_{K} / \mathfrak{q}\right|=\ell^{f_{\mathfrak{q}}},
$$

où $f_{\mathfrak{q}}$ est le degré résiduel de q. On suppose que $E$ a mauvaise réduction additive en $\mathfrak{q}$ avec potentiellement bonne réduction. Alors, pour tout nombre premier $p \geq 3$ tel que $p \neq \ell$, l'action de $I_{\mathfrak{q}}$, sous-groupe d'inertie en $\mathfrak{q}$, sur $E[p]$ se fait par l'intermédiaire d'un certain quotient fini $\Phi_{\mathfrak{q}}$ de $I_{\mathfrak{q}}$ ([ST68] ) :

$$
I_{\mathfrak{q}} \longrightarrow \Phi_{\mathfrak{q}} \hookrightarrow \operatorname{Aut}(E[p]) .
$$

On a alors les deux résultats suivants. Ceux-ci sont connus pour $K=\mathbf{Q}$ et ont été utilisés par Serre dans [Ser72, §5] pour traiter des exemples numériques.

Proposition 1.1 On suppose que le groupe $\Phi_{\mathfrak{q}}$ n'est pas cyclique. Alors, la représentation $\rho_{p}$ est irréductible pour tout nombre premier $p \geq 5$.

Proposition 1.2 On suppose que pour tout entier $n \geq 0$, l'ordre du groupe $\Phi_{\mathfrak{q}}$ ne divise pas $N(\mathfrak{q})^{n}(N(\mathfrak{q})-1)$. Alors, la représentation $\rho_{p}$ est irréductible pour tout nombre premier $p \geq 3$ tel que $p \neq \ell$.

Remarque. Si $\ell \geq 5$, on peut remplacer l'hypothèse par : l'ordre du groupe $\Phi_{\mathfrak{q}}$ ne divise pas $N(\mathfrak{q})-1$.

Comme corollaires des propositions ci-dessus, on obtient les résultats suivants dans le cas où $\mathfrak{q}$ divise 2 ou 3 . 
Corollaire 1.3 On suppose que $\mathfrak{q}$ divise 2 et que l'une des conditions suivantes est satisfaite :

1. le groupe $\Phi_{\mathfrak{q}}$ est d'ordre 8 ou 24 ;

2. le groupe $\Phi_{\mathfrak{q}}$ est d'ordre 3 ou 6 et le degré résiduel $f_{\mathfrak{q}}$ est impair.

Alors, la représentation $\rho_{p}$ est irréductible pour tout nombre premier $p \geq 5$.

Lorsque $\mathfrak{q}$ divise 2, l'étude faite dans [Bil09] permet parfois de calculer l'ordre du groupe $\Phi_{\mathfrak{q}}$ directement à partir de la valuation de l'invariant modulaire de $E$ ([Bil09, th. 1]). Si $K$ est une extension quadratique de $\mathbf{Q}$ (ou plus généralement si le degré sur $\mathbf{Q}_{2}$ du complété de $K$ en $\mathfrak{q}$ est $\leq 2$ ), le théorème [Bil09, th. 2] et Cal04 fournissent en toute généralité l'ordre du groupe $\Phi_{\mathfrak{q}}$ en fonction des coefficients d'une équation de Weierstrass de $E$.

Remarque. La condition de parité dans le corollaire précédent est nécessaire. En effet, soient $K$ l'extension de $\mathbf{Q}$ engendrée par une racine du polynôme

$$
\left(X^{2}+5 X+1\right)^{3}\left(X^{2}+13 X+49\right)-\frac{2^{4} \cdot 13^{3}}{3^{2}} X
$$

et $E$ la courbe elliptique définie sur $K$ par l'équation

$$
y^{2}=x^{3}-x^{2}-4 x-4 .
$$

Alors, le degré résiduel de $K$ en l'unique idéal $\mathfrak{p}_{2}$ de $\mathcal{O}_{K}$ divisant 2 , est $f_{\mathfrak{p}_{2}}=2$ et la courbe $E$ a un défaut de semi-stabilité d'ordre 6 en $\mathfrak{p}_{2}$. Pour autant la représentation $\rho_{7}: \mathrm{G}_{K} \longrightarrow \mathrm{GL}_{2}\left(\mathbf{F}_{7}\right)$ est réductible car $K$ correspond au souscorps de $\overline{\mathbf{Q}}$ laissé fixe par le stablilisateur dans $\operatorname{Gal}(\overline{\mathbf{Q}} / \mathbf{Q})$ d'un sous-groupe d'ordre 7 de $E(\overline{\mathbf{Q}})$ ([KO92, p. 273]).

Lorsque $\mathfrak{q}$ divise 3 , on a le corollaire suivant.

Corollaire 1.4 On suppose que $\mathfrak{q}$ divise 3 et que l'une des conditions suivantes est satisfaite :

1. le groupe $\Phi_{\mathfrak{q}}$ est d'ordre 12 ;

2. le groupe $\Phi_{\mathfrak{q}}$ est d'ordre 4 et le degré résiduel $f_{\mathfrak{q}}$ est impair.

Alors, la représentation $\rho_{p}$ est irréductible pour tout nombre premier $p \geq 5$.

\section{Rappels}

Dans toute cette section, on fixe un corps de nombres $K$ contenu dans $\overline{\mathbf{Q}}$ et une courbe elliptique $E$ définie sur $K$. Soit $p$ un nombre premier exceptionnel. Le groupe $E[p]$ possède alors une droite $D$ stable par $\mathrm{G}_{K}$. Notons $\lambda$ le caractère donnant l'action de $\mathrm{G}_{K}$ sur $D$. On l'appelle caractère d'isogénie associé à $D$. Dans une base convenable de $E[p]$ sur $\mathbf{F}_{p}$, la représentation $\rho_{p}$ est représentable matriciellement par

$$
\left(\begin{array}{cc}
\lambda & * \\
0 & \lambda^{\prime}
\end{array}\right)
$$

où $\lambda$ et $\lambda^{\prime}$ s'interprètent comme des caractères de $\mathrm{G}_{K}$ à valeurs dans $\mathbf{F}_{p}^{*}$. On a

$$
\operatorname{det} \rho_{p}=\lambda \cdot \lambda^{\prime}=\chi_{p},
$$


où $\chi_{p}$ est le caractère donnant l'action de $\mathrm{G}_{K}$ sur les racines $p$-ièmes de l'unité (caractère cyclotomique).

La représentation $\rho_{p}$ se factorise à travers le groupe de Galois de l'extension $K(E[p]) / K$, où $K(E[p])$ est le corps engendré sur $K$ par les coordonnées des points de $p$-torsion de $E$. On note encore $\rho_{p}, \lambda, \lambda^{\prime}$ et $\chi_{p}$ les morphismes passés au quotient.

Soit $\mathfrak{q}$ est un idéal premier de $\mathcal{O}_{K}$. On note $I_{\mathfrak{q}}$ un sous-groupe d'inertie en $\mathfrak{q}$ de $\operatorname{Gal}(K(E[p]) / K)$. Si $E$ a bonne réduction en $\mathfrak{q}$ et $\mathfrak{q}$ ne divise pas $p$, l'extension $K(E[p]) / K$ est non ramifiée en $\mathfrak{q}$ par le critère de Néron-Ogg-Shafarevich. On note $\sigma_{\mathfrak{q}}$ une subsitution de Frobenius en $\mathfrak{q}$ de $\operatorname{Gal}(K(E[p]) / K$ ) (bien définie à conjugaison près).

Le résultat suivant est bien connu (c.f. [Sil92, Th.2.4]) et intervient de façon cruciale dans la démonstration des théorèmes 1 et 2

Proposition 2.1 (Hasse - Weil) Soit $\mathfrak{q}$ est un idéal premier de $\mathcal{O}_{K}$ en lequel $E$ a bonne réduction. Les racines complexes de $P_{\mathfrak{q}}$ sont de module $N(\mathfrak{q})^{1 / 2}$. En particulier, on a

$$
\left|t_{\mathfrak{q}}\right| \leq 2 N(\mathfrak{q})^{1 / 2} .
$$

Si de plus $\mathfrak{q}$ ne divise pas $p$, le polynôme caractéristique de $\rho_{p}\left(\sigma_{\mathfrak{q}}\right)$ est $\overline{P_{\mathfrak{q}}}=P_{\mathfrak{q}}$ $(\bmod p) \in \mathbf{F}_{p}[X]$. En particulier, on a

$$
\overline{P_{\mathfrak{q}}}\left(\lambda\left(\sigma_{\mathfrak{q}}\right)\right)=0 .
$$

\subsection{L'ensemble $\operatorname{Exc}(E / K)$}

L'objectif de ce $\S$ est de démontrer le résultat suivant.

Proposition 2.2 Les conditions suivantes sont équivalentes :

1. la courbe $E$ n'a pas de multiplication complexe sur $K\left(\right.$ i.e. $\left.\operatorname{End}_{K}(E)=\mathbf{Z}\right)$;

2. l'ensemble $\operatorname{Exc}(E / K)$ est fini.

Démonstration. L'implication 1) $\Rightarrow 2$ résulte du théorème de Šafarevič sur la finitude des classes de $K$-isomorphisme de courbes elliptiques $K$-isogènes à une courbe donnée ([Sil92, IX §6]). Elle est due à Serre et démontrée dans [Ser68, IV-9].

Réciproquement, si $E$ a des multiplications complexes sur $K$ (i.e. $\operatorname{End}_{K}(E)$ est de rang 2 comme $\mathbf{Z}$-module), alors

$$
\operatorname{End}_{K}(E) \otimes \mathbf{Q}=\operatorname{End}_{\overline{\mathbf{Q}}}(E) \otimes \mathbf{Q}
$$

et $K$ contient le corps quadratique imaginaire $L=\operatorname{End}_{K}(E) \otimes \mathbf{Q}$. Soit $p$ un nombre premier décomposé dans $L$. On a

$$
p \mathcal{O}_{L}=\pi \cdot \bar{\pi},
$$

où $\mathcal{O}_{L}$ est l'anneau des entiers de $L$. Alors, l'ensemble $E[\pi]$ des points de $E$ annulés par les éléments de $\pi$ est défini sur $K$ et d'ordre $p$ ([Lan87, ch.9 §4]). On en déduit que l'ensemble $\operatorname{Exc}(E / K)$ est infini. 


\subsection{Ramification et caractère d'isogénie}

On suppose que $p$ est un nombre premier exceptionnel pour $E$. Le résultat suivant se déduit de l'étude de la restriction de $\rho_{p}$ aux sous-groupes d'inertie de $\operatorname{Gal}(K(E[p]) / K)$ telle qu'elle est faite, par exemple, dans [Ser68, IV], [Ser72, $\S \S 1.11-1.12$ ] et [Kra97] (voir également [Dav08, §1] pour une discussion similaire).

Proposition 2.3 Supposons $p \geq 5$ non ramifié dans $K$.

1. Le caractère $\lambda^{12}$ est non ramifié en dehors des idéaux premiers de $\mathcal{O}_{K}$ divisant $p$.

2. Soit $\mathfrak{p}$ un idéal de $\mathcal{O}_{K}$ divisant $p$. On suppose que E n'a pas mauvaise réduction additive en $\mathfrak{p}$ avec potentiellement bonne réduction de hauteur 2 (supersingulière). Alors, il existe un entier $\alpha_{\mathfrak{p}} \in\{0,12\}$ tel que

$$
\left.\lambda^{12}\right|_{I_{\mathfrak{p}}}=\left.\chi_{p}^{\alpha_{\mathfrak{p}}}\right|_{I_{\mathfrak{p}}} .
$$

Remarques.

1. Dans une base convenable, la représentation sur les points de $p$-torsion de la courbe $E / D$ est représentable matriciellement par

$$
\left(\begin{array}{ll}
\lambda^{\prime} & * \\
0 & \lambda
\end{array}\right)
$$

Autrement dit, d'après l'égalité (2), on peut toujours, si on le souhaite, remplacer la famille $\left\{\alpha_{\mathfrak{p}}\right\}_{\mathfrak{p} \mid p}$ par la famille $\left\{12-\alpha_{\mathfrak{p}}\right\}_{\mathfrak{p} \mid p}$.

2. On peut montrer en utilisant la description locale de $\rho_{p}$ donnée dans la proposition [Kra97, prop.2] que si $\mathfrak{p}$ divise $p$ et $E$ a mauvaise réduction additive en $\mathfrak{p}$ avec potentiellement bonne réduction supersingulière, alors il existe un entier $\alpha_{\mathfrak{p}} \in\{4,6,8\}$ tel que

$$
\left.\lambda^{12}\right|_{I_{\mathfrak{p}}}=\left.\chi_{p}^{\alpha_{\mathfrak{p}}}\right|_{I_{\mathfrak{p}}} .
$$

3. Dans sa thèse ([Dav08] $)$, A. David démontre que si $K$ ne contient pas le corps de classes de Hilbert d'un corps quadratique imaginaire, il existe alors une constante effective $C(K)$, ne dépendant que de $K$ (et donc pas de $E$ ) telle que si $p>C(K)$, on a $\alpha_{\mathfrak{p}}=6$ pour tout idéal premier $\mathfrak{p}$ de $\mathcal{O}_{K}$ divisant $p$ (voir également Mom95). Nous n'utiliserons pas ces résultats.

\subsection{Théorie du corps de classes et caractère d'isogénie}

On reprend les hypothèses et notations précédentes. En particulier, $p$ est un nombre premier $\geq 5$ non ramifié dans $K$ et on suppose que pour tout idéal premier $\mathfrak{p}$ de $\mathcal{O}_{K}$ divisant $p, E$ n'a pas mauvaise réduction additive en $\mathfrak{p}$ avec potentiellement bonne réduction de hauteur 2. Étant donné un idéal premier $\mathfrak{p}$ de $\mathcal{O}_{K}$ au-dessus de $p$, on désigne par

$$
N_{\mathfrak{p}}:\left(\mathcal{O}_{K} / \mathfrak{p}\right)^{*} \longrightarrow \mathbf{F}_{p}^{*}
$$

le morphisme norme. L'objectif de ce paragraphe 2.3 est de démontrer la proposition ci-dessous, cruciale dans la démonstration des th. 1 et 2 . Elle figure également sous une forme légèrement différente dans la thèse de David ([Dav08, prop. 2.2.1]) ainsi que dans l'article [Mom95, lem. 1] de Momose (sous l'hypothèse que $K / \mathbf{Q}$ est galoisienne). 
Proposition 2.4 Soit $a \in \mathcal{O}_{K}$ premier à $p$ et $a \mathcal{O}_{K}=\prod_{\mathfrak{q}} \mathfrak{q}^{v_{\mathfrak{q}}(a)}$ la décomposition de $a \mathcal{O}_{K}$ en produit d'idéaux premiers de $\mathcal{O}_{K}$. On suppose que pour tout idéal premier $\mathfrak{q}$ de $\mathcal{O}_{K}$ divisant a, E a bonne réduction en $\mathfrak{q}$. Alors, on a :

$$
\prod_{\mathfrak{q} \mid a} \lambda\left(\sigma_{\mathfrak{q}}\right)^{12 v_{\mathfrak{q}}(a)}=\prod_{\mathfrak{p} \mid p} N_{\mathfrak{p}}(a+\mathfrak{p})^{\alpha_{\mathfrak{p}}}
$$

où $\alpha_{\mathfrak{p}} \in\{0,12\}$ est défini à la proposition 2.3.

\subsubsection{Un lemme de la théorie du corps de classes.}

Soient $L$ l'extension de $K$ trivialisant le caractère $\lambda^{12}$ et $\mu_{p}$ le groupe de racines $p$-ièmes de l'unité dans $\overline{\mathbf{Q}}$. D'après l'accouplement de Weil, on a $\mu_{p} \subset$ $K(E[p])$. Donc $L\left(\mu_{p}\right)$ est une sous-extension abélienne de $K(E[p]) / K$. On note $I_{K}$ le groupe des idèles de $K$ et

$$
r: I_{K} \longrightarrow \operatorname{Gal}\left(L\left(\mu_{p}\right) / K\right),
$$

le morphisme de réciprocité global donné par la théorie du corps de classes. Il est surjectif et son noyau contient les idèles principales.

Soit $v$ une place de $K$. On note $K_{v}$ le complété de $K$ en $v$ et on identifie $K$ à un sous-corps de $K_{v}$. On désigne par

$$
r_{v}: K_{v}^{*} \hookrightarrow I_{K} \longrightarrow \operatorname{Gal}\left(L\left(\mu_{p}\right) / K\right)
$$

la composée de l'injection de $K_{v}^{*}$ dans $I_{K}$ par le morphisme de réciprocité global.

Si $\mathfrak{q}$ est un idéal premier de $\mathcal{O}_{K}$ de bonne réduction ne divisant pas $p$, on rappelle que l'extension $K(E[p]) / K$ est non ramifiée en $\mathfrak{q}$. La restriction à $\operatorname{Gal}\left(L\left(\mu_{p}\right) / K\right)$ d'une substitution de Frobenius en $\mathfrak{q}$ du groupe $\operatorname{Gal}(K(E[p]) / K)$ (bien définie à conjugaison près) est unique. On la note encore $\sigma_{\mathfrak{q}}$. De même, on note encore $\chi_{p}$ (resp. $\lambda$ ) la restriction du caractère cyclotomique (resp. d'isogénie) à $\operatorname{Gal}\left(L\left(\mu_{p}\right) / K\right)$.

Le lemme suivant regroupe plusieurs résultats classiques de la théorie du corps de classes qui seront utiles à la démonstration de la proposition 2.4. La démonstration du troisième point est tirée de [Kra07, App. 1 prop. 1].

Lemme 2.1 Soit $v$ une place de $K$.

1. Si $v$ est une place infinie de $K$, on a $\lambda^{12}\left(r_{v}(a)\right)=1$.

2. $S i v=\mathfrak{q}$ est une place finie de $K$ ne divisant pas $p$, on a $r_{\mathfrak{q}}\left(\mathcal{U}_{\mathfrak{q}}\right)=\{1\}$, où $\mathcal{U}_{\mathfrak{q}}$ est le groupe des unités de l'anneau d'entiers du corps $K_{\mathfrak{q}}$. Si de plus, $\mathfrak{q}$ divise a, alors $r_{\mathfrak{q}}\left(\pi_{\mathfrak{q}}\right)=\sigma_{\mathfrak{q}}$, où $\pi_{\mathfrak{q}}$ est une uniformisante de $K_{\mathfrak{q}}$.

3. Si $v=\mathfrak{p}$ est une place finie de $K$ divisant $p$, alors $r_{\mathfrak{p}}(a)$ appartient au sous-groupe d'inertie en $\mathfrak{p}$ de $L\left(\mu_{p}\right) / K$ et on a

$$
\chi_{p}\left(r_{\mathfrak{p}}(a)\right)=N_{\mathfrak{p}}(a+\mathfrak{p})^{-1}
$$

Démonstration. Soit $v$ une place de $K$. On distingue trois cas.

1. Supposons que $v$ soit une place infinie de $K$. Soit $L^{\prime}$ l'extension de $K$ trivialisant le caractère $\lambda$,

$$
r^{\prime}: I_{K} \longrightarrow \operatorname{Gal}\left(L^{\prime}\left(\mu_{p}\right) / K\right)
$$


le morphisme de réciprocité global donné par la théorie du corps de classes et

$$
r_{v}^{\prime}: K_{v}^{*} \hookrightarrow I_{K} \stackrel{r^{\prime}}{\longrightarrow} \operatorname{Gal}\left(L^{\prime}\left(\mu_{p}\right) / K\right) .
$$

L'image de l'application $r_{v}^{\prime}$ est d'ordre $\leq 2$. Par ailleurs, l'image par $\lambda^{12}$ d'un élément de $\operatorname{Gal}\left(L^{\prime}\left(\mu_{p}\right) / K\right)$ ne dépend que de sa restriction à $\operatorname{Gal}\left(L\left(\mu_{p}\right) / K\right)$. D'où :

$$
\lambda^{12}\left(r_{v}(a)\right)=\lambda^{12}\left(r_{v}^{\prime}(a)\right)
$$

puis

$$
\lambda\left(r_{v}^{\prime}(a)\right)^{12}=\lambda\left(r_{v}^{\prime}(a)^{12}\right)=1
$$

D'où le résultat.

2. Supposons que $v=\mathfrak{q}$ soit une place finie de $K$ ne divisant pas $p$. Alors, d'après [Neu86, l'image par $r_{\mathfrak{q}}$ de $\mathcal{U}_{\mathfrak{q}}$ est un sous-groupe d'inertie en $\mathfrak{q}$ de l'extension $L\left(\mu_{p}\right) / K$. Or celle-ci est non ramifiée en $\mathfrak{q}$ d'après le critère de Néron-Ogg-Šafarevič. D'où l'égalité

$$
r_{\mathfrak{q}}\left(\mathcal{U}_{\mathfrak{q}}\right)=\{1\} .
$$

Si de plus $\mathfrak{q}$ divise $a$ alors $E$ a bonne réduction en $\mathfrak{q}$ par hypothèse et d'après [Neu86, l'image par $r_{\mathfrak{q}}$ de $\pi_{\mathfrak{q}}$ est la substitution de Frobenius en $\mathfrak{q}$ de l'extension $L\left(\mu_{p}\right) / K$. Autrement dit, $r_{\mathfrak{q}}\left(\pi_{\mathfrak{q}}\right)=\sigma_{\mathfrak{q}}$.

3. Supposons que $v=\mathfrak{p}$ soit une place finie de $K$ divisant $p$. On note $\overline{\mathbf{Q}_{p}}$ une clôture algébrique de $\mathbf{Q}_{p}$. Comme $p$ est non ramifié dans $K$, on identifie $K_{\mathfrak{p}}$ à l'extension non ramifiée de $\mathbf{Q}_{p}$ contenue dans $\overline{\mathbf{Q}_{p}}$ dont le degré sur $\mathbf{Q}_{p}$ est le degré résiduel de $\mathfrak{p}$ sur $p$. On note $K^{a b}$ la clôture abélienne de $K$ dans $\overline{\mathbf{Q}}, K_{\mathfrak{p}}^{a b}$ la clôture abélienne de $K_{\mathfrak{p}}$ dans $\overline{\mathbf{Q}_{p}}$,

$$
\Theta_{\mathfrak{p}}: K_{\mathfrak{p}}^{*} \longrightarrow \operatorname{Gal}\left(K_{\mathfrak{p}}^{a b} / K_{\mathfrak{p}}\right)
$$

le morphisme de réciprocité local en $\mathfrak{p}$ et

$$
\operatorname{Res}_{\mathfrak{p}}: \operatorname{Gal}\left(K_{\mathfrak{p}}^{a b} / K_{\mathfrak{p}}\right) \longrightarrow \operatorname{Gal}\left(L\left(\mu_{p}\right) / K\right)
$$

le morphisme de restriction. D'après la compatibilité entre la théorie du corps de classes locale et globale, on a, pour tout $x \in K_{\mathfrak{p}}^{*}$,

$$
\operatorname{Res}_{\mathfrak{p}}\left(\Theta_{\mathfrak{p}}(x)\right)=r_{\mathfrak{p}}(x) .
$$

Or, d'après le corollaire de [Kra07, App. 1 prop. 1], on a

$$
\Theta_{\mathfrak{p}}(a)(\zeta)=\zeta^{n^{-1}}
$$

où $\zeta$ est une racine primitive $p$-ième de l'unité dans $\overline{\mathbf{Q}_{p}}$ et $n$ est un entier tel que

$$
N_{\mathfrak{p}}(a+\mathfrak{p})=n \quad(\bmod p \mathbf{Z}) .
$$

D'où le résultat voulu, d'après l'égalité (3).

Cela termine la démonstration du lemme 2.1. 


\subsubsection{Démonstration de la proposition 2.4.}

L'entier $a$ est non nul car premier à $p$. L'image par le morphisme de réciprocité global de l'idèle principale $(a)_{v}$ est triviale :

$$
\prod_{v} r_{v}(a)=1
$$

Si $v$ est une place infinie de $K$, alors d'après le lemme 2.1, on a

$$
\lambda^{12}\left(r_{v}(a)\right)=1
$$

Si $v=\mathfrak{q}$ est une place finie de $K$ ne divisant ni $p$, ni $a$, alors $a \in \mathcal{U}_{K_{\mathfrak{q}}}$. D'après loc. cit. on a donc $r_{\mathfrak{q}}(a)=1$.

Si $v=\mathfrak{q}$ est une place finie de $K$ divisant $a$. Alors, $a=u \cdot \pi_{\mathfrak{q}}^{v_{\mathfrak{q}}(a)}$, où $\pi_{\mathfrak{q}}$ est une uniformisante de $K_{\mathfrak{q}}$ et $u \in \mathcal{U}_{K_{\mathfrak{q}}}$. D'après loc. cit. on a donc $r_{\mathfrak{q}}(a)=\sigma_{\mathfrak{q}}^{v_{\mathfrak{q}}}(a)$, puis

$$
\lambda^{12}\left(r_{\mathfrak{q}}(a)\right)=\left(\lambda^{12}\left(\sigma_{\mathfrak{q}}\right)\right)^{v_{\mathfrak{q}}(a)}=\lambda\left(\sigma_{\mathfrak{q}}\right)^{12 v_{\mathfrak{q}}(a)} .
$$

Si $v=\mathfrak{p}$ est une place finie de $K$ divisant $p$, alors d'après loc. cit., $r_{\mathfrak{p}}(a)$ appartient au sous-groupe d'inertie en $\mathfrak{p}$ de $L\left(\mu_{p}\right) / K$ et on a

$$
\chi_{p}\left(r_{\mathfrak{p}}(a)\right)=N_{\mathfrak{p}}(a+\mathfrak{p})^{-1}
$$

Or, d'après la proposition 2.3, on a

$$
\left.\lambda^{12}\right|_{I_{\mathfrak{p}}}=\left.\chi_{p}^{\alpha_{\mathfrak{p}}}\right|_{I_{\mathfrak{p}}} .
$$

On en déduit que l'on a

$$
\lambda^{12}\left(r_{\mathfrak{p}}(a)\right)=N_{\mathfrak{p}}(a+\mathfrak{p})^{-\alpha_{\mathfrak{p}}} .
$$

D'après les égalités (4)-(7) ci-dessus, on a

$$
\begin{aligned}
1 & =\prod_{v} \lambda^{12}\left(r_{v}(a)\right) \\
& =\prod_{\mathfrak{q} \mid a} \lambda\left(\sigma_{\mathfrak{q}}\right)^{12 v_{\mathfrak{q}}(a)} \cdot \prod_{\mathfrak{p} \mid p} N_{\mathfrak{p}}(a+\mathfrak{p})^{-\alpha_{\mathfrak{p}}} .
\end{aligned}
$$

Cela démontre la proposition 2.4

\section{Démonstrations des théorèmes 1 et 2}

Soient $K$ un corps de nombres contenu dans $\overline{\mathbf{Q}}, E$ une courbe elliptique définie sur $K$ et $p$ un nombre premier exceptionnel pour $(E, K)$. On suppose $p \geq 5$, non ramifié dans $K$ et pour tout idéal premier $\mathfrak{p}$ de $\mathcal{O}_{K}$ divisant $p, E$ n'a pas mauvaise réduction additive en $\mathfrak{p}$ avec potentiellement bonne réduction de hauteur 2 .

Avant de démontrer les théorèmes 1 et 2, on commence par définir pour tout anneau intègre $A$, une loi de monoïde commutatif $*$ sur un sous-ensemble de $A[X]$ et par en étudier les propriétés utiles. 


\subsection{Loi de monoïde}

Soit $A$ un anneau intègre de corps des fractions $L$ et $\bar{L}$ une clôture algébrique de $L$. On note $M_{A}$ le sous-ensemble de $A[X]$ constitué des polynômes unitaires ne s'annulant pas en 0 .

Lemme 3.1 L'application

$$
\begin{aligned}
M_{A} \times M_{A} & \longrightarrow A[X] \\
(P, Q) & \longmapsto(P * Q)(X)=\operatorname{Res}_{Z}\left(P(Z), Q(X / Z) Z^{\operatorname{deg} Q}\right)
\end{aligned}
$$

a une image contenue dans $M_{A}$. Elle définit une loi de monö̈de commutatif sur $M_{A}$ d'élément neutre $\Psi_{1}(X)=X-1$. De plus, si $P, Q \in M_{A}$ s'écrivent

$$
P(X)=\prod_{i=1}^{n}\left(X-\alpha_{i}\right) \quad \text { et } \quad Q(X)=\prod_{j=1}^{m}\left(X-\beta_{j}\right)
$$

dans $\bar{L}[X]$, on a

$$
(P * Q)(X)=\prod_{\substack{1 \leq i \leq n \\ 1 \leq j \leq m}}\left(X-\alpha_{i} \beta_{j}\right) .
$$

En particulier,

$$
(P * Q)(0)=(-1)^{\operatorname{deg} P \cdot \operatorname{deg} Q} P(0)^{\operatorname{deg} Q} Q(0)^{\operatorname{deg} P} .
$$

Démonstration. Il s'agit de vérifier que pour tout $P, Q$ et $R \in M_{A}$, on a

1. $P * Q \in M_{A}$;

2. $P * \Psi_{1}=\Psi_{1} * P=P$;

3. $(P * Q) * R=P *(Q * R)$;

4. $P * Q=Q * P$.

On suppose que le polynôme $Q$ s'écrit

$$
Q(X)=X^{m}+b_{m-1} X^{m-1}+\cdots+b_{1} X+b_{0}, \quad \text { avec } b_{0} \neq 0 .
$$

Alors,

$$
Q\left(\frac{X}{Z}\right) Z^{m}=b_{0} Z^{m}+b_{1} X Z^{m-1}+\cdots+b_{m-1} X^{m-1} Z+X^{m} \in A[X][Z]
$$

et $\operatorname{deg}_{Z}\left(Q(X / Z) Z^{m}\right)=m=\operatorname{deg} Q\left(\right.$ car $\left.b_{0} \neq 0\right)$. Par définition du résultant de deux polynômes ([Bou81, A IV.71 §6 Déf. 1]), on a donc $P * Q \in A[X]$. Par ailleurs, sur $\bar{L}$, on a

$$
Q\left(\frac{X}{Z}\right) Z^{m}=Q(0) \prod_{j=1}^{m}\left(Z-\frac{1}{\beta_{j}} X\right)
$$

et d'après Bou81, A IV.75 §6 Cor. 1],

$$
(P * Q)(X)=Q(0)^{n} \prod_{i, j}\left(\alpha_{i}-\frac{1}{\beta_{j}} X\right) .
$$


Or, $Q(0)=\prod_{j=1}^{m}\left(-\beta_{j}\right)$, donc

$$
(P * Q)(X)=\prod_{i=1}^{n} \prod_{j=1}^{m}\left(X-\alpha_{i} \beta_{j}\right) .
$$

C'est la formule de l'énoncé. On en déduit que l'on a :

$$
\begin{aligned}
& -(P * Q)(0)=(-1)^{\operatorname{deg} P \cdot \operatorname{deg} Q} P(0)^{\operatorname{deg} Q} Q(0)^{\operatorname{deg} P} \neq 0, \text { donc } P * Q \in M_{A} ; \\
& -P * \Psi_{1}=\Psi_{1} * P=P ; \\
& -P * Q=Q * P
\end{aligned}
$$

De plus, les polynômes $(P * Q) * R$ et $P *(Q * R)$ ont les mêmes racines dans $\bar{L}$ comptées avec multiplicités. Comme ils sont unitaires, ils sont égaux. D'où le lemme.

Lemme 3.2 Soient $r \geq 1$ et $P \in M_{A}$. Il existe un unique polynôme $P^{(r)} \in M_{A}$ tel que

$$
P^{(r)}\left(X^{r}\right)=\left(P * \Psi_{r}\right)(X)
$$

où $\Psi_{r}(X)=X^{r}-1$. L'application $P \mapsto P^{(r)}$ est un morphisme de monö̈des pour la loi $*$. De plus, si $P \in M_{A}$ se factorise sur $\bar{L}$ de la façon suivante

$$
P(X)=\prod_{i=1}^{n}\left(X-\alpha_{i}\right), \quad \text { on a } \quad P^{(r)}(X)=\prod_{i=1}^{n}\left(X-\alpha_{i}^{r}\right) .
$$

Démonstration. Soit $P \in M_{A}$. L'unicité d'un polynôme $P^{(r)}$ vérifiant la relation (8) est immédiate. Posons

$$
P(X)=\prod_{i=1}^{n}\left(X-\alpha_{i}\right) \quad \text { avec } \alpha_{i} \in \bar{L}
$$

et $\zeta_{r}$ une racine $r$-ième de l'unité dans $\bar{L}$. Vérifions alors qu'il existe bien un polynôme de $M_{A}$ satisfaisant à l'égalité (8) :

$$
\begin{aligned}
\left(P * \Psi_{r}\right)(X)= & \operatorname{Res}_{Z}\left(P(Z), X^{r}-Z^{r}\right) \\
= & (-1)^{n} \prod_{i=1}^{n} \prod_{k=0}^{r-1}\left(\alpha_{i}-\zeta_{r}^{k} X\right) \\
& \text { d'après [Bou81, A IV.75 §6 Cor. 1] } \\
= & (-1)^{n} \prod_{i=1}^{n}(-1)^{r} \zeta_{r}^{\frac{r(r-1)}{2}} \prod_{k=0}^{r-1}\left(X-\zeta_{r}^{-k} \alpha_{i}\right)
\end{aligned}
$$

Or, on a $\zeta_{r}^{\frac{r(r-1)}{2}}=(-1)^{r+1}$. D'où

$$
\left(P * \Psi_{r}\right)(X)=\prod_{i=1}^{n} \prod_{k=0}^{r-1}\left(X-\zeta_{r}^{-k} \alpha_{i}\right)=\prod_{i=1}^{n}\left(X^{r}-\alpha_{i}^{r}\right) .
$$

Autrement dit,

$$
\left(P * \Psi_{r}\right)(X)=P^{(r)}\left(X^{r}\right)
$$


où l'on a posé

$$
P^{(r)}(X)=\prod_{i=1}^{n}\left(X-\alpha_{i}^{r}\right) .
$$

Cela démontre qu'il existe bien un polynôme $P^{(r)}$ de $A[X]$ satisfaisant à l'égalité (81) et qu'il est donné par la formule (91). Par ailleurs, d'après le lemme 3.1. on a $P^{(r)}(0)=(-1)^{(r+1) \operatorname{deg} P} P(0)^{r} \neq 0$ et comme $P^{(r)}$ est unitaire, on a $P^{(r)} \in M_{A}$. On en déduit que l'application $P \mapsto P^{(r)}$ est bien définie.

Vérifions enfin qu'il s'agit bien d'un morphisme de monoïdes. On a $\Psi_{1}^{(r)}=$ $\Psi_{1}$. Soient $P$ et $Q$ dans $M_{A}$. D'après le lemme 3.1 et la formule (9), les polynômes $(P * Q)^{(r)}$ et $P^{(r)} * Q^{(r)}$ ont les mêmes racines dans $\bar{L}$ comptées avec multiplicités. Ils sont donc égaux. D'où le lemme 3.2

Lemme 3.3 Soient $A$ et $B$ deux anneaux intègres et $\varphi: A \rightarrow B$ un morphisme d'anneaux. L'ensemble

$$
M_{A}^{\varphi}=\left\{P \in M_{A} \mid \varphi(P(0)) \neq 0\right\}
$$

est stable pour la loi $*$. L'application $\varphi$ induit un morphisme de monoïdes (encore noté $\varphi$ )

$$
\varphi: M_{A}^{\varphi} \longrightarrow M_{B}
$$

Soient $P \in M_{A}^{\varphi}$ et $r \geq 1$. Alors, $P^{(r)} \in M_{A}^{\varphi}$ et on $a(\varphi(P))^{(r)}=\varphi\left(P^{(r)}\right)$.

Démonstration. D'après le lemme 3.1] si $P, Q \in M_{A}^{\varphi} \subset M_{A}$, on a $P * Q \in M_{A}$ et

$$
(P * Q)(0)=(-1)^{\operatorname{deg} P \cdot \operatorname{deg} Q} P(0)^{\operatorname{deg} Q} Q(0)^{\operatorname{deg} P} .
$$

D'où

$$
\varphi((P * Q)(0))=(-1)^{\operatorname{deg} P \cdot \operatorname{deg} Q} \varphi(P(0))^{\operatorname{deg} Q} \varphi(Q(0))^{\operatorname{deg} P} \neq 0
$$

$\operatorname{car} \varphi(P(0)) \neq 0, \varphi(Q(0)) \neq 0$ et $B$ est intègre. Donc $M_{A}^{\varphi}$ est bien un sousensemble de $M_{A}$ stable pour la loi $*$. On a $\varphi\left(\Psi_{1}\right)=\Psi_{1}$ et la relation

$$
\varphi(P * Q)=\varphi(P) * \varphi(Q), \quad \text { pour } P, Q \in M_{A}^{\varphi},
$$

résulte de la définition du résultant de deux polynômes ([Bou81, A IV.72 §6]) en termes de déterminant de Sylvester.

Soient $P \in M_{A}^{\varphi}$ et $r \geq 1$. Alors, d'après le lemme 3.2, on a $P^{(r)} \in M_{A}$ et

$$
\varphi\left(P^{(r)}(0)\right)=(-1)^{(r+1) \operatorname{deg} P} \varphi(P(0))^{r} \neq 0
$$

$\operatorname{car} \varphi(P(0)) \neq 0$ et $B$ est intègre. D'où $P^{(r)} \in M_{A}^{\varphi}$. De plus, d'après la formule (8) et la définition du résultant de deux polynômes (Bou81, A IV.72 §6]), on a

$$
\varphi\left(P^{(r)}\left(X^{r}\right)\right)=\varphi\left(\left(P * \Psi_{r}\right)(X)\right)=\left(\varphi(P) * \Psi_{r}\right)(X)=\varphi(P)^{(r)}\left(X^{r}\right) .
$$

D'où l'égalité $(\varphi(P))^{(r)}=\varphi\left(P^{(r)}\right)$ et le lemme 3.3 .

Remarque. D'après le lemme ci-dessus, l'application de réduction $\varphi: \mathbf{Z} \rightarrow \mathbf{Z} / p \mathbf{Z}$ induit un morphisme de monoïdes

$$
\begin{aligned}
M_{\mathbf{Z}}^{\varphi} & \longrightarrow M_{\mathbf{F}_{p}} \\
P & \longmapsto
\end{aligned}
$$

En particulier, $\overline{P * Q}=\bar{P} * \bar{Q}$ pour tout $P, Q \in M_{\mathbf{Z}}^{\varphi}$. 


\subsection{Démonstration du théorème 1}

Soit $\ell$ un nombre premier tel que $E$ ait bonne réduction en tout idéal premier de $\mathcal{O}_{K}$ divisant $\ell$. Il s'agit de montrer que $p$ divise $B_{\ell}$.

\subsubsection{Le polynôme $P_{\ell}^{*}$}

Soit $\prod_{\mathfrak{q} \mid \ell} \mathfrak{q}^{v_{\mathfrak{q}}(\ell)}$ la décomposition de l'idéal $\ell \mathcal{O}_{K}$ en produit d'idéaux premiers de $\mathcal{O}_{K}$. On note $g_{\ell}$ le cardinal de l'ensemble $\{\mathfrak{q} \mid \ell\}$. On rappelle que $E$ a par hypothèse bonne réduction en tout idéal premier $\mathfrak{q}$ de $\mathcal{O}_{K}$ divisant $\ell$. Le polynôme $P_{\ell}^{*}$ donné par la formule (1) est alors bien défini et à coefficients entiers.

Lemme 3.4 Le polynôme $P_{\ell}^{*}$ appartient à $M_{\mathbf{Z}}$ et vérifie :

$$
P_{\ell}^{*}(0)=\ell^{12 \cdot d \cdot 2^{g_{\ell}-1}} .
$$

Ses racines complexes sont de module $\ell^{6 d}$. Si de plus $\ell \neq p$, alors $P_{\ell}^{*} \in M_{\mathbf{Z}}^{\varphi}$ et on a

$$
\overline{P_{\ell}^{*}}(\Omega)=0, \quad \text { où } \quad \Omega=\prod_{\mathfrak{q} \mid \ell} \lambda\left(\sigma_{\mathfrak{q}}\right)^{12 v_{\mathfrak{q}}(\ell)} \in \mathbf{F}_{p} .
$$

Démonstration. Pour tout $\mathfrak{q} \mid \ell$, le polynôme $P_{\mathfrak{q}}$ est unitaire, à coefficients entiers et on a (prop. 2.1) :

$$
P_{\mathfrak{q}}(0)=N(\mathfrak{q})=\ell^{f_{\mathfrak{q}}} .
$$

En particulier, $P_{\mathfrak{q}} \in M_{\mathbf{Z}}$. D'après les lemmes 3.1 et 3.2 , le polynôme $P_{\ell}^{*}$ est bien défini (la loi $*$ est associative) et indépendant de l'ordre des idéaux premiers dans la décomposition de $\ell$ dans $K$ (la loi $*$ est commutative). De plus, $P_{\ell}^{*}$ appartient à $M_{\mathbf{Z}} \subset \mathbf{Z}[X]$.

Soient $P_{1}, \ldots, P_{n} \in M_{A}$ de degrés respectifs $d_{1}, \ldots, d_{n}$. On montre par récurrence sur $n$, à partir de la formule pour $n=2 \mathrm{du}$ lemme 3.1 que l'on a

$$
\left(P_{1} * \cdots * P_{n}\right)(0)=(-1)^{(n+1) d_{1} \cdots d_{n}} \prod_{i=1}^{n} P_{i}(0)^{\Pi_{j \neq i} d_{j}} .
$$

De plus, d'après le lemme 3.2 , pour tout $P \in M_{\mathbf{Z}}$ et tout entier $r \geq 1$, on a

$$
P^{(r)}(0)=(-1)^{(r+1) \operatorname{deg} P} P(0)^{r} .
$$

Comme pour tout idéal $\mathfrak{q} \mid \ell$, on a $\operatorname{deg} P_{\mathfrak{q}}=2$, on en déduit

$$
P_{\ell}^{*}(0)=\prod_{\mathfrak{q} \mid \ell} P_{\mathfrak{q}}(0)^{12 v_{\mathfrak{q}}(\ell) \cdot 2^{g_{\ell}-1}}=\prod_{\mathfrak{q} \mid \ell}\left(\ell^{f_{\mathfrak{q}}}\right)^{12 v_{\mathfrak{q}}(\ell) \cdot 2^{g_{\ell}-1}}=\ell^{12 \cdot 2^{g_{\ell}-1} \sum_{\mathfrak{q} \mid \ell} f_{\mathfrak{q}} v_{\mathfrak{q}}(\ell)} .
$$

D'où la formule car $\sum_{\mathfrak{q} \mid \ell} f_{\mathfrak{q}} v_{\mathfrak{q}}(\ell)=d$.

Par ailleurs, d'après la proposition 2.1. les racines complexes de $P_{\mathfrak{q}}$ sont de module $N(\mathfrak{q})^{1 / 2}=\ell^{f_{\mathfrak{q}} / 2}$. Donc, d'après le lemme 3.2 celles de $P_{\mathfrak{q}}^{\left(12 v_{\mathfrak{q}}(\ell)\right)}$ sont de module $\ell^{6 f_{\mathfrak{q}} v_{\mathfrak{q}}(\ell)}$. D'après le lemme 3.1, celles de $P_{\ell}^{*}$ sont de module

$$
\prod_{\mathfrak{q} \mid \ell} \ell^{6 f_{\mathfrak{q}} v_{\mathfrak{q}}(\ell)}=\ell^{6 \sum_{\mathfrak{q} \mid \ell} f_{\mathfrak{q}} v_{\mathfrak{q}}(\ell)}=\ell^{6 d} .
$$


Supposons à présent $\ell \neq p$. Alors, d'après la formule (10), on a $P_{\ell}^{*} \in M_{\mathbf{Z}}^{\varphi}$. D'après la proposition 2.1, on a

$$
\overline{P_{\mathfrak{q}}}\left(\lambda\left(\sigma_{\mathfrak{q}}\right)\right)=0 .
$$

Donc d'après le lemme 3.2 , on a :

$$
{\overline{P_{\mathfrak{q}}}}^{\left(12 v_{\mathfrak{q}}(\ell)\right)}\left(\lambda\left(\sigma_{\mathfrak{q}}\right)^{12 v_{\mathfrak{q}}(\ell)}\right)=0 \quad(\bmod p) .
$$

Puis,

$$
\begin{aligned}
\overline{P_{\ell}^{*}}(\Omega) & =\overline{\mathfrak{q} \mid \ell} \overline{P_{\mathfrak{q}}^{\left(12 v_{\mathfrak{q}}(\ell)\right)}}\left(\prod_{\mathfrak{q} \mid \ell} \lambda\left(\sigma_{\mathfrak{q}}\right)^{12 v_{\mathfrak{q}}(\ell)}\right) \\
& =\left(\underset{\mathfrak{q} \mid \ell}{*}{\overline{P_{\mathfrak{q}}}}^{\left(12 v_{\mathfrak{q}}(\ell)\right)}\right)\left(\prod_{\mathfrak{q} \mid \ell} \lambda\left(\sigma_{\mathfrak{q}}\right)^{12 v_{\mathfrak{q}}(\ell)}\right) \quad(\text { lemme 3.3) } \\
& =0 \quad(\bmod p) \quad(\text { d'après le lemme 3.1 et la relation (11) }) .
\end{aligned}
$$

D'où le lemme 3.4

\subsubsection{Fin de la démonstration du théorème 1}

Supposons $p=\ell$. Alors, pour $d \geq 2$, par définition de $B_{p}^{(d)}$, il existe un entier $k>0$ tel que $P_{p}^{*}\left(p^{12 k}\right)$ divise $B_{p}^{(d)}$. D'où $p$ divise $B_{p}^{(d)}$ car d'après le lemme 3.4 :

$$
P_{p}^{*}\left(p^{12 k}\right) \equiv P_{p}^{*}(0) \equiv 0 \quad(\bmod p) .
$$

Supposons $p \neq \ell$. D'après la proposition 2.4 appliquée à $a=\ell$, on a :

$$
\Omega=\prod_{\mathfrak{q} \mid \ell} \lambda\left(\sigma_{\mathfrak{q}}\right)^{12 v_{\mathfrak{q}}(\ell)}=\prod_{\mathfrak{p} \mid p} N_{\mathfrak{p}}(\ell+\mathfrak{p})^{\alpha_{\mathfrak{p}}} .
$$

Or, par définition on a

$$
N_{\mathfrak{p}}(\ell+\mathfrak{p})=\ell^{1+p+\cdots+p^{f_{\mathfrak{p}}-1}} \quad(\bmod p)=\ell^{f_{\mathfrak{p}}} \quad(\bmod p)
$$

où $N(\mathfrak{p})=\left|\mathcal{O}_{K} / \mathfrak{p}\right|=\ell^{f_{\mathfrak{p}}}$. D'où

$$
\prod_{\mathfrak{p} \mid p} N_{\mathfrak{p}}(\ell+\mathfrak{p})^{\alpha_{\mathfrak{p}}}=\ell^{\sum_{\mathfrak{p} \mid p} f_{\mathfrak{p}} \alpha_{\mathfrak{p}}} .
$$

Or, $\alpha_{\mathfrak{p}} \in\{0,12\}$ d'après la proposition 2.3 et on pose

$$
k=\sum_{\substack{\mathfrak{p} \mid p \\ \alpha_{\mathfrak{p}}=12}} f_{\mathfrak{p}} \geq 0
$$

de sorte que

$$
\sum_{\mathfrak{p} \mid p} f_{\mathfrak{p}} \alpha_{\mathfrak{p}}=12 k .
$$

Comme $p$ est non ramifié dans $K$, on a

$$
d=\sum_{\mathfrak{p} \mid p} f_{\mathfrak{p}} .
$$


Or, d'après la remarque 2.2 suivant la proposition 2.3 on peut toujours, si on le souhaite, remplacer la famille $\left\{\alpha_{\mathfrak{p}}\right\}_{\mathfrak{p} \mid p}$ par la famille $\left\{12-\alpha_{\mathfrak{p}}\right\}_{\mathfrak{p} \mid p}$, donc on peut supposer que l'on a :

$$
\sum_{\mathfrak{p} \mid p} f_{\mathfrak{p}} \alpha_{\mathfrak{p}} \leq \sum_{\mathfrak{p} \mid p} f_{\mathfrak{p}}\left(12-\alpha_{\mathfrak{p}}\right) .
$$

Autrement dit, d'après les égalités (14) et (15)

$$
12 k \leq 12(d-k)
$$

soit encore

$$
k \leq\left[\frac{d}{2}\right] .
$$

D'après les égalités (13) et (14) on a

$$
\prod_{\mathfrak{p} \mid p} N_{\mathfrak{p}}(\ell+\mathfrak{p})^{\alpha_{\mathfrak{p}}}=\ell^{12 k} \quad(\bmod p) .
$$

Par ailleurs, d'après le lemme 3.4, on a $\overline{P_{\ell}^{*}}(\Omega)=0$. Donc, d'après les égalités (12) et (16), il vient $\overline{P_{\ell}^{*}}\left(\ell^{12 k}\right)=0(\bmod p)$, c'est-à-dire

$$
P_{\ell}^{*}\left(\ell^{12 k}\right) \equiv 0 \quad(\bmod p) .
$$

D'où le théorème 1 .

\subsection{Démonstration du théorème 2}

Soit $\mathfrak{q}$ un idéal premier de bonne réduction, $\gamma_{\mathfrak{q}}$ un générateur de $\mathfrak{q}^{h}$ et $\mathfrak{m}_{\gamma_{\mathfrak{q}}}$ son polynôme minimal sur $\mathbf{Q}$. On commence par un lemme préliminaire.

Lemme 3.5 Le polynôme $\mathfrak{m}_{\gamma_{\mathfrak{q}}}^{(12)}$ appartient à $M_{\mathbf{Z}}$ et vérifie pour $\mathfrak{p}$ divisant $p$

$$
\overline{\left(\mathfrak{m}_{\gamma_{\mathfrak{q}}}^{(12)}\right)^{* f_{\mathfrak{p}}}}\left(N_{\mathfrak{p}}\left(\gamma_{\mathfrak{q}}+\mathfrak{p}\right)^{12}\right)=0 \quad(\bmod p) .
$$

Démonstration. Le polynôme $\mathfrak{m}_{\gamma_{\mathfrak{q}}}$ est irréductible et unitaire. Il appartient donc à $M_{\mathbf{Z}}$ et il en va de même pour $\mathfrak{m}_{\gamma_{q}}^{(12)}$ d'après le lemme 3.2. Par définition, on a

$$
N_{\mathfrak{p}}\left(\gamma_{\mathfrak{q}}+\mathfrak{p}\right)=\gamma_{\mathfrak{q}} \cdot \gamma_{\mathfrak{q}}^{p} \cdots \gamma_{\mathfrak{q}}^{p^{f_{\mathfrak{p}}-1}}+\mathfrak{p} \in \mathbf{Z} / p \mathbf{Z}
$$

et

$$
\overline{\mathfrak{m}_{\gamma_{\mathfrak{q}}}}\left(\gamma_{\mathfrak{q}}+\mathfrak{p}\right)=0 \quad(\bmod \mathfrak{p}) .
$$

Or, le polynôme $\mathfrak{m}_{\gamma_{\mathrm{q}}}$ est à coefficients dans $\mathbf{Z}$, d'où $\overline{\mathfrak{m}_{\gamma_{\mathfrak{q}}}^{(p)}}=\overline{\mathfrak{m}_{\gamma_{\mathfrak{q}}}}$. On en déduit donc avec les lemmes 3.1 et 3.2 que l'on a :

$$
\begin{array}{cccc}
\overline{\mathfrak{m}_{\gamma_{\mathfrak{q}}}^{(12)}}\left(\gamma_{\mathfrak{q}}^{12}+\mathfrak{p}\right) & = & 0 & (\bmod \mathfrak{p}) \\
\vdots & & & \vdots \\
\overline{\mathfrak{m}_{\gamma_{\mathfrak{q}}}^{(12)}}\left(\gamma_{\mathfrak{q}}^{12 p^{f_{\mathfrak{p}}-1}}+\mathfrak{p}\right) & = & 0 & (\bmod \mathfrak{p}) .
\end{array}
$$


Puis avec le lemme 3.3, il vient

$$
\begin{aligned}
& \overline{\left(\mathfrak{m}_{\gamma_{\mathfrak{q}}}^{(12)}\right)^{* f_{\mathfrak{p}}}}\left(N_{\mathfrak{p}}\left(\gamma_{\mathfrak{q}}+\mathfrak{p}\right)^{12}\right) \\
& ={\overline{\mathfrak{m}_{\gamma_{\mathfrak{q}}}^{(12)}}}^{* f_{\mathfrak{p}}}\left(\gamma_{\mathfrak{q}}^{12} \cdot \gamma_{\mathfrak{q}}^{12 p} \cdots \gamma_{\mathfrak{q}}^{12 p^{f_{\mathfrak{p}}-1}}+\mathfrak{p}\right) \\
& =0(\bmod p)
\end{aligned}
$$

$\operatorname{car} N_{\mathfrak{p}}\left(\gamma_{\mathfrak{q}}+\mathfrak{p}\right) \in \mathbf{Z} / p \mathbf{Z}$. D'où le lemme.

Démontrons à présent le théorème 2, Supposons que q divise $p$. Alors, 0 est une racine commune de $P_{\mathfrak{q}}^{(12 h)}$ et $\mathfrak{m}_{\gamma_{\mathfrak{q}}}^{(12)}$ modulo $p$. Donc $p$ divise l'entier $\operatorname{Res}\left(P_{\mathfrak{q}}^{(12 h)}, \mathfrak{m}_{\gamma_{\mathfrak{q}}}^{(12)}\right)$ et par suite, si $d \geq 2, p$ divise $R_{\mathfrak{q}}$.

Supposons que $\mathfrak{q}$ ne divise pas $p$. Alors, d'après la proposition 2.4 appliquée à $a=\gamma_{\mathfrak{q}}$, on a

$$
\lambda\left(\sigma_{\mathfrak{q}}\right)^{12 h}=\prod_{\mathfrak{p} \mid p} N_{\mathfrak{p}}\left(\gamma_{\mathfrak{q}}+\mathfrak{p}\right)^{\alpha_{\mathfrak{p}}}=\prod_{\substack{\mathfrak{p} \mid p \\ \alpha_{\mathfrak{p}=12}}} N_{\mathfrak{p}}\left(\gamma_{\mathfrak{q}}+\mathfrak{p}\right)^{12}
$$

Or, d'après le lemme 3.5, on a

$$
\overline{\left(\mathfrak{m}_{\gamma_{\mathfrak{q}}}^{(12)}\right)^{* f_{\mathfrak{p}}}}\left(N_{\mathfrak{p}}\left(\gamma_{\mathfrak{q}}+\mathfrak{p}\right)^{12}\right)=0 \quad(\bmod p) .
$$

On en déduit donc avec le lemme 3.1 que l'on a

$$
\underset{\substack{\mathfrak{p} \mid p \\ \alpha_{\mathfrak{p}=12}}}{*\left(\mathfrak{m}_{\gamma_{\mathfrak{q}}}^{(12)}\right)^{* f_{\mathfrak{p}}}}\left(\prod_{\substack{\mathfrak{p} \mid p \\ \alpha_{\mathfrak{p}=12}}} N_{\mathfrak{p}}\left(\gamma_{\mathfrak{q}}+\mathfrak{p}\right)^{12}\right)=0 \quad(\bmod p),
$$

puis avec l'égalité (17) ci-dessus et le lemme 3.3

$$
\overline{\left(\mathfrak{m}_{\gamma_{\mathfrak{q}}}^{(12)}\right)^{* k}}\left(\lambda\left(\sigma_{\mathfrak{q}}\right)^{12 h}\right)=0 \quad(\bmod p),
$$

où l'on a posé

$$
k=\sum_{\substack{\mathfrak{p} \mid p \\ \alpha \mathfrak{p}=12}} f_{\mathfrak{p}} \geq 0 .
$$

Comme au 3.2 .2 on peut supposer $k \leq[d / 2]$. Par ailleurs, $\lambda\left(\sigma_{\mathfrak{q}}\right)^{12 h}$ est une racine de $P_{\mathfrak{q}}^{(12 h)}(\bmod p)$. On en déduit donc que $p$ divise $\operatorname{Res}\left(P_{\mathfrak{q}}^{(12 h)},\left(\mathfrak{m}_{\gamma_{\mathfrak{q}}}^{(12)}\right)^{* k}\right)$ et par suite, $p$ divise $R_{\mathfrak{q}}$. Cela démontre la première partie du théorème 2 . Il reste à voir que si $E$ est sans multiplication complexe sur $\overline{\mathbf{Q}}$, alors $R_{\mathfrak{q}} \neq 0$ pour une infinité de $\mathfrak{q}$.

Supposons $R_{\mathfrak{q}}=0$. Alors, il existe une racine complexe $\alpha_{\mathfrak{q}}$ de $P_{\mathfrak{q}}$ telle que $\alpha_{\mathfrak{q}}^{12 h}$ soit racine de $\left(\mathfrak{m}_{\gamma_{\mathfrak{q}}}^{(12)}\right)^{* k}$ pour un certain entier $0 \leq k \leq[d / 2]$. C'est impossible pour $k=0$ car $\alpha_{\mathfrak{q}}^{12 h} \neq 1$. On a donc $k \geq 1$ (et par suite $d \geq 2$ ) et $\alpha_{\mathfrak{q}}^{12 h}$ s'écrit comme un produit de $k$ conjugués de $\gamma_{\mathfrak{q}}$ élevés à la puissance 12 . Notons $L^{\mathfrak{q}}$ le corps engendré par $\alpha_{\mathfrak{q}}$. C'est une extension de degré au plus 2 de $\mathbf{Q}$. On distingue deux cas : 
1. soit $\alpha_{\mathfrak{q}}^{12 h} \notin \mathbf{Q}$ et alors $L^{\mathfrak{q}}=\mathbf{Q}\left(\alpha_{\mathfrak{q}}^{12 h}\right)$ est inclus dans $K^{\text {gal }}$, la clôture galoisienne de $K$ dans $\overline{\mathbf{Q}}$; en particulier, $L^{\mathfrak{q}}$ est non ramifié en dehors des premiers divisant $D_{K}$.

2. Soit $\alpha_{\mathfrak{q}}^{12 h} \in \mathbf{Q}$ et alors

$$
\zeta=\frac{\overline{\alpha_{\mathfrak{q}}}}{\alpha_{\mathfrak{q}}}
$$

est une racine $12 h$-ième de l'unité contenue dans $L^{\mathfrak{q}}$. C'est donc une racine primitive 2-ième, 3-ième, 4-ième ou 6-ième de l'unité et l'on a :

(a) soit $\zeta=1, t_{\mathfrak{q}}^{2}=4 N(\mathfrak{q})$ et $L^{\mathfrak{q}}=\mathbf{Q}$;

(b) soit $\zeta=-1, t_{\mathfrak{q}}=0$ et $L^{\mathfrak{q}}=\mathbf{Q}(\sqrt{-1})$ ou $\mathbf{Q}(\sqrt{-\ell})$;

(c) soit $\zeta=j$ ou $j^{2}\left(\right.$ avec $\left.j^{2}+j+1=0\right), t_{\mathfrak{q}}^{2}=N(\mathfrak{q})$, donc $f_{\mathfrak{q}}$ est pair et $L^{\mathfrak{q}}=\mathbf{Q}(\sqrt{-3})$

(d) soit $\zeta=i$ ou $-i$ (avec $\left.i^{2}=-1\right), t_{\mathfrak{q}}^{2}=2 N(\mathfrak{q})$, donc $\ell=2$ et $f_{\mathfrak{q}}$ est impair. On en déduit $L^{\mathfrak{q}}=\mathbf{Q}(\sqrt{-1})$;

(e) soit $\zeta=-j$ ou $-j^{2}, t_{\mathfrak{q}}^{2}=3 N(\mathfrak{q})$, donc $\ell=3$ et $f_{\mathfrak{q}}$ est impair. On en déduit et $L^{\mathfrak{q}}=\mathbf{Q}(\sqrt{-3})$.

On en déduit que dans ce cas la courbe $E$ a réduction supersingulière en

$\mathfrak{q}$ et que le corps $L^{\mathfrak{q}}$ est non ramifié en dehors de $\{2,3, \ell\}$.

Autrement dit, on a montré que si $R_{\mathfrak{q}}=0$, alors le corps $L^{\mathfrak{q}}$ est non ramifié en dehors des nombres premiers divisant $6 \ell D_{K}$. Or, d'après un résultat de Serre ([Ser68, IV-14(d)]), on sait que si $E$ est sans multiplication complexe sur $\overline{\mathbf{Q}}$, alors pour tout ensemble fini $P$ de nombres premiers, il existe une infinité d'idéaux premiers $\mathfrak{q}$ tels que $L^{\mathfrak{q}}$ soit ramifié en tout nombre premier appartenant à $P$. Compte-tenu de l'étude précédente, on en déduit qu'il existe une infinité d'idéaux premiers $\mathfrak{q}$ pour lesquels on a $R_{\mathfrak{q}} \neq 0$. Cela achève la démonstration du théorème 2

\section{Bornes uniformes}

\subsection{Démonstration de la proposition 1.1}

Soit $\mathfrak{q}$ un idéal premier de $\mathcal{O}_{K}$ tel que le sous-groupe $\Phi_{\mathfrak{q}}$ soit non cyclique. Compte-tenu de la structure des groupes $\Phi$, l'idéal premier $\mathfrak{q}$ a nécessairement caractéristique résiduelle $\ell=2$ ou 3 ([Ser72, $\S 5.6(a)]$ ) et $\left|\Phi_{\mathfrak{q}}\right|=8$ ou 24 (resp. 12) si $\ell=2($ resp. $\ell=3)$. L'irréductibilité de $\rho_{p}$ résulte alors du lemme 4.1 cidessous et du fait que $\Phi_{\mathfrak{q}}$ se plonge dans $\operatorname{Aut}(E[p])(\operatorname{car} \ell \neq p$ et $p \geq 3$, Ser72, $\S 5.6(a)])$.

Notation 1 On rappelle qu'un sous-groupe maximal de Aut $(E[p])$ stabilisant une droite de $E[p]$ est appelé sous-groupe de Borel.

Lemme 4.1 Soit $H$ un sous-groupe de $\operatorname{Gal}(K(E[p]) / K)$. On suppose $H$ non abélien fini. Si $p$ ne divise pas l'ordre de $H$, alors $H$ ne se plonge pas dans un sous-groupe de Borel de $\operatorname{Aut}(E[p])$. 
Démonstration. Supposons qu'il existe un morphisme injectif $\iota$ de $G$ dans un sous-groupe de Borel $B$ de $\operatorname{Aut}(E[p])$. Dans une base convenable de $E[p]$ sur $\mathbf{F}_{p}, B$ est représentable matriciellement par le Borel standard

$$
\left(\begin{array}{ll}
* & * \\
0 & *
\end{array}\right)
$$

Il contient alors le sous-groupe $S$ d'ordre $p$ engendré par l'élément

$$
u=\left(\begin{array}{ll}
1 & 1 \\
0 & 1
\end{array}\right)
$$

C'est un sous-groupe distingué de $B$. Comme l'ordre de $H$ est premier à $p$, le morphisme composé

$$
H \stackrel{\iota}{\hookrightarrow} B \rightarrow B / S
$$

est injectif. Par ailleurs, $B / S$ est abélien. D'où une contradiction car $H$ est supposé non abélien.

\subsection{Démonstration de la proposition 1.2}

Soient $p \geq 3$ un nombre premier exceptionnel et $\mathfrak{q}$ un idéal premier de $\mathcal{O}_{K}$ de caractéristique résiduelle $\ell \neq p$ en lequel $E$ a mauvaise réduction additive avec potentiellement bonne réduction. On souhaite montrer qu'il existe un entier $n \geq 0$ tel que l'ordre du groupe $\Phi_{\mathfrak{q}}$ divise $N(\mathfrak{q})^{n}(N(\mathfrak{q})-1)$.

$\mathrm{Vu}$ la théorie du corps de classes, le caractère $\lambda$ s'interprète comme un homomorphisme

$$
\lambda: \operatorname{Gal}\left(K^{\mathfrak{m}} / K\right) \longrightarrow \mathbf{F}_{p}^{*},
$$

où $\mathfrak{m}$ est le conducteur de $\lambda$ et $K^{\mathfrak{m}}$ le corps de classes de rayon $\mathfrak{m}$. Alors, le caractère $\lambda$ est ramifié en $\mathfrak{q}$ (cf. [Ser72, $\S \S 1.12$ et 5.6]) et on a une factorisation du type

$$
\mathfrak{m}=\mathfrak{m}^{\prime} \cdot \mathfrak{q}^{n+1}, \quad \text { où } n \geq 0 \quad \text { et }\left(\mathfrak{m}^{\prime}, \mathfrak{q}\right)=1 .
$$

L'ordre du groupe $\Phi_{\mathfrak{q}}$ divise l'indice de ramification en $\mathfrak{q}$ de l'extension $K^{\mathfrak{m}} / K$. Or l'extension intermédiaire $K^{\mathfrak{m}^{\prime}} / K$ est non ramifiée en $\mathfrak{q}$. Donc l'ordre de $\Phi_{\mathfrak{q}}$ divise le cardinal du groupe $\operatorname{Gal}\left(K^{\mathfrak{m}} / K^{\mathfrak{m}^{\prime}}\right)$. Notons $h_{\mathfrak{m}}$ (resp. $\left.h_{\mathfrak{m}^{\prime}}\right)$ le cardinal du groupe $\operatorname{Gal}\left(K^{\mathfrak{m}} / K\right)$ (resp. $\left.\operatorname{Gal}\left(K^{\mathfrak{m}^{\prime}} / K\right)\right)$. Alors, d'après [Coh00, cor.3.2.4], on a

$$
\left|\operatorname{Gal}\left(K^{\mathfrak{m}} / K^{\mathfrak{m}^{\prime}}\right)\right|=\frac{h_{\mathfrak{m}}}{h_{\mathfrak{m}^{\prime}}}=\frac{\left(\mathcal{U}: \mathcal{U}_{\mathfrak{m}^{\prime}, 1}\right)}{\left(\mathcal{U}: \mathcal{U}_{\mathfrak{m}, 1}\right)} N(\mathfrak{q})^{n}(N(\mathfrak{q})-1),
$$

où $\mathcal{U}_{\mathfrak{m}, 1}\left(\right.$ resp. $\left.\mathcal{U}_{\mathfrak{m}^{\prime}, 1}\right)$ désigne le sous-groupe du groupe des unités $\mathcal{U}$ de $\mathcal{O}_{K}$ qui sont congrues à 1 modulo $\mathfrak{m}$ (resp. $\mathfrak{m}^{\prime}$ ) au sens de [Coh00, Def.3.2.2]. Or, comme $\mathfrak{m}^{\prime}$ divise $\mathfrak{m}$, l'indice de $\mathcal{U}_{\mathfrak{m}^{\prime}, 1}$ dans $\mathcal{U}$ divise celui de $\mathcal{U}_{\mathfrak{m}, 1}$. Donc, l'ordre de $\operatorname{Gal}\left(K^{\mathfrak{m}} / K^{\mathfrak{m}^{\prime}}\right)$ divise $N(\mathfrak{q})^{n}(N(\mathfrak{q})-1)$ et il en va de même en particulier pour l'ordre de $\Phi_{\mathfrak{q}}$. D'où la proposition 1.2 .

Remarque. Lorsque $\ell \geq 5$, on a $\left|\Phi_{\mathfrak{q}}\right|=2,3,4$ ou 6 (Ser72, p. 312]). Or $N(\mathfrak{q})$ est premier à 12 , donc $\left|\Phi_{\mathfrak{q}}\right|$ divise $N(\mathfrak{q})^{n}(N(\mathfrak{q})-1)$ pour un certain entier $n$ si et seulement si $\left|\Phi_{\mathfrak{q}}\right|$ divise $N(\mathfrak{q})-1$. Cela justifie la remarque 1.2 . 


\subsection{Démonstration des corollaires 1.3 et 1.4}

Supposons que $\mathfrak{q}$ divise 2 . Lorsque $\left|\Phi_{\mathfrak{q}}\right|=8$ ou 24 , le groupe $\Phi_{\mathfrak{q}}$ n'est pas abélien ([Ser72, 5.6(a)]) et la conclusion résulte de la prop. 1.1. Pour $\left|\Phi_{\mathfrak{q}}\right|=3$ ou 6, supposons la représentation $\rho_{p}$ réductible. Alors, d'après la prop. 1.2. l'ordre de $\Phi_{\mathfrak{q}}$ divise $2^{f_{\mathfrak{q}}}\left(2^{f_{\mathfrak{q}}}-1\right)$. Or, $2^{f_{\mathfrak{q}}}-1 \equiv 1(\bmod 3)$ car $f_{\mathfrak{q}}$ est impair. D'où une contradiction et le corollaire 1.3 .

Supposons que $\mathfrak{q}$ divise 3 . Lorsque $\left|\Phi_{\mathfrak{q}}\right|=12$, le groupe $\Phi_{\mathfrak{q}}$ n'est pas abélien ([Ser72, 5.6(a)]) et la conclusion résulte comme ci-dessus de la prop. 1.1. Pour $\left|\Phi_{\mathfrak{q}}\right|=4$, supposons la représentation $\rho_{p}$ réductible. Alors, d'après la prop. 1.2, l'ordre de $\Phi_{\mathfrak{q}}$ divise $3^{f_{\mathfrak{q}}}\left(3^{f_{\mathfrak{q}}}-1\right)$. Or, $3^{f_{\mathfrak{q}}}-1 \equiv 2(\bmod 4)$ car $f_{\mathfrak{q}}$ est impair, d'où une contradiction et le corollaire 1.4 .

\section{Exemples numériques}

L'objet de cette section est de déterminer explicitement, pour certaines courbes elliptiques $E$ définies sur des corps de nombres $K$, l'ensemble $\operatorname{Exc}(E / K)$ et d'illustrer ainsi chacun des résultats du §1]

\subsection{Stratégie}

On traite successivement des courbes définies sur des corps quadratiques, puis une sur un corps cubique et enfin une dernière sur un corps biquadratique. Pour la plupart d'entre elles, on commence par déterminer son type de réduction en chaque idéal premier. Si pour l'un d'entre eux, on est dans un cas d'application des « résultats uniformes» (prop. 1.1 et 1.2 et cor. 1.3 et 1.4) du \$1 il ne reste plus alors à traiter que le cas $p=2$ et éventuellement $p=3$ et $p=\ell$ où $\ell$ est un nombre premier $\geq 5$. Sinon, on applique le critère du théorème 1 . On cherche alors un nombre premier $\ell$ de bonne réduction pour lequel $B_{\ell}$ soit non nul. Si $d$ est impair, c'est automatique (cor. 1.2). Si $d$ est pair, l'existence d'un tel nombre premier n'est malheureusement pas garantie comme le montre l'exemple 5.8. Cependant dans le cas quadratique $(d=2)$, cela ne pose aucun problème d'en trouver un dans la pratique.

Après quelques itérations, on obtient alors un ensemble très restreint de nombres premiers contenant 2, 3 et les premiers ramifiés dans le corps. On traite ensuite «à la main » ceux qui restent. Soit on trouve un idéal premier $\mathfrak{q}$ de bonne réduction ne divisant pas $p$ tel que $P_{\mathfrak{q}}$ soit irréductible modulo $p$ et alors $p$ n'est pas exceptionnel, soit on montre que $E$ possède un sous-groupe stable d'ordre $p$ et alors $p$ est exceptionnel.

\subsection{Notations}

La courbe $E$ est donnée sous forme d'une équation de Weierstrass

$$
y^{2}+a_{1} x y+a_{3} y=x^{3}+a_{2} x^{2}+a_{4} x+a_{6},
$$

avec $a_{i} \in \mathcal{O}_{K}$. On adopte les notations standard de Tate ([Tat75]). Pour chaque idéal premier $\mathfrak{p}$ de $\mathcal{O}_{K}$, on note $v_{\mathfrak{p}}$ la valuation en $\mathfrak{p}$ de $K$ normalisée par $v_{\mathfrak{p}}\left(K^{*}\right)=\mathbf{Z}$. 
Étant donné un nombre premier $\ell$, on note $\mathfrak{S}_{\ell}^{(d)}$ l'ensemble des diviseurs premiers de $B_{\ell}$. Lorsque $E$ est définie sur un corps quadratique, on dispose de deux programmes pari, disponibles à l'adresse :

$$
\text { http://people.math.jussieu.fr/ billerey/. }
$$

appelés, Trace0fFrobenius et ExceptionalPrimes, permettant respectivement de calculer la famille $\left\{t_{\mathfrak{q}}\right\}_{\mathfrak{q} \mid \ell}$ et de déterminer l'ensemble $\mathfrak{S}_{\ell}^{(2)}$ (et aussi l'entier $\left.B_{\ell}\right)$.

\subsection{Les polynômes $P_{\ell}^{*}$ dans le cas quadratique}

On suppose que $K$ est un corps quadratique, i.e. $d=2$. Pour un $\ell$ de bonne réduction, on a alors $B_{\ell}=P_{\ell}^{*}(1) \cdot P_{\ell}^{*}\left(\ell^{12}\right)$ et $P_{\ell}^{*}(1) \neq 0$ (lemme 3.4). La proposition suivante donne une description explicite des polynômes $P_{\ell}^{*}$ et de la condition $B_{\ell}=0$. On rappelle au préalable que pour tout entier $n \geq 1$, il existe un unique polynôme $T_{n}$ appartenant à $\mathbf{Z}[X]$ tel que pour tout nombre réel $\theta$, on ait $T_{n}(\cos \theta)=\cos (n \theta)$. Le polynôme $T_{n}$ s'appelle le $n$-ième polynôme de Tchebychev (de première espèce). On a en particulier,

$$
\begin{aligned}
T_{12}(X) & =2048 X^{12}-6144 X^{10}+6912 X^{8}-3584 X^{6}+840 X^{4}-72 X^{2}+1 \\
\text { et } T_{24}(X) & =2 T_{12}(X)^{2}-1 .
\end{aligned}
$$

Proposition 5.1 On suppose K/Q quadratique. Soit $\ell$ nombre premier de bonne réduction. On est dans l'une des situations suivantes.

1. Soit $\ell$ est ramifié dans $K, \ell \mathcal{O}_{K}=\mathfrak{q}^{2}$ et on a

$$
P_{\ell}^{*}(X)=P_{\mathfrak{q}}^{(24)}(X)=X^{2}-2 \ell^{12} T_{24}\left(t_{\mathfrak{q}} / 2 \sqrt{\ell}\right) X+\ell^{24} .
$$

En particulier, on a

$$
P_{\ell}^{*}\left(\ell^{12}\right)=-\ell^{12} t_{\mathfrak{q}}^{2}\left(t_{\mathfrak{q}}^{2}-\ell\right)^{2}\left(t_{\mathfrak{q}}^{2}-4 \ell\right)\left(t_{\mathfrak{q}}^{2}-2 \ell\right)^{2}\left(t_{\mathfrak{q}}^{2}-3 \ell\right)^{2}\left(t_{\mathfrak{q}}^{4}-4 \ell t_{\mathfrak{q}}^{2}+\ell^{2}\right)^{2} .
$$

Ainsi $P_{\ell}^{*}\left(\ell^{12}\right)=0$ si et seulement si $t_{\mathfrak{q}} \equiv 0(\bmod \ell)$, c'est-à-dire si et seulement si $E$ a bonne réduction supersingulière en $\mathfrak{q}$.

2. Soit $\ell$ est inerte dans $K, \ell \mathcal{O}_{K}=\mathfrak{q}$ et on a

$$
P_{\ell}^{*}(X)=P_{\mathfrak{q}}^{(12)}(X)=X^{2}-2 \ell^{12} T_{12}\left(t_{\mathfrak{q}} / 2 \ell\right) X+\ell^{24} .
$$

En particulier, on a

$$
P_{\ell}^{*}\left(\ell^{12}\right)=-\ell^{12} t_{\mathfrak{q}}^{2}\left(t_{\mathfrak{q}}^{2}-\ell^{2}\right)^{2}\left(t_{\mathfrak{q}}^{2}-4 \ell^{2}\right)\left(t_{\mathfrak{q}}^{2}-3 \ell^{2}\right)^{2} .
$$

Ainsi $P_{\ell}^{*}\left(\ell^{12}\right)=0$ si et seulement si $t_{\mathfrak{q}} \equiv 0(\bmod \ell)$, c'est-à-dire si et seulement si $E$ a bonne réduction supersingulière en $\mathfrak{q}$.

3. Soit $\ell$ est décomposé dans $K, \ell \mathcal{O}_{K}=\mathfrak{q}_{1} \mathfrak{q}_{2}$ et on a

$$
\begin{aligned}
P_{\ell}^{*}(X)=\left(P_{\mathfrak{q}_{1}} * P_{\mathfrak{q}_{2}}\right)^{(12)}(X) & =X^{4}-4 \ell^{12} T_{12}\left(t_{\mathfrak{q}_{1}} / 2 \sqrt{\ell}\right) T_{12}\left(t_{\mathfrak{q}_{2}} / 2 \sqrt{\ell}\right) X^{3} \\
-2 \ell^{24}\left(1-2\left(T_{12}\left(t_{\mathfrak{q}_{1}} / 2 \sqrt{\ell}\right)^{2}+T_{12}\left(t_{\mathfrak{q}_{2}} / 2 \sqrt{\ell}\right)^{2}\right)\right) X^{2} & \\
& -4 \ell^{36} T_{12}\left(t_{\mathfrak{q}_{1}} / 2 \sqrt{\ell}\right) T_{12}\left(t_{\mathfrak{q}_{2}} / 2 \sqrt{\ell}\right) X+\ell^{48}
\end{aligned}
$$


En particulier, on a

$$
\begin{aligned}
P_{\ell}^{*}\left(\ell^{12}\right)=\ell^{36}\left(t_{\mathfrak{q}_{1}}^{2}-t_{\mathfrak{q}_{2}}^{2}\right)^{2}\left(\left(t_{\mathfrak{q}_{1}}^{2}+t_{\mathfrak{q}_{2}}^{2}\right.\right. & \left.-3 \ell)^{2}-t_{\mathfrak{q}_{1}}^{2} t_{\mathfrak{q}_{2}}^{2}\right)^{2}\left(t_{\mathfrak{q}_{1}}^{2}+t_{\mathfrak{q}_{2}}^{2}-4 \ell\right)^{2} \\
& \times\left(\left(t_{\mathfrak{q}_{1}}^{2}+t_{\mathfrak{q}_{2}}^{2}-\ell\right)^{2}-3 t_{\mathfrak{q}_{1}}^{2} t_{\mathfrak{q}_{2}}^{2}\right)^{2}
\end{aligned}
$$

Ainsi $P_{\ell}^{*}\left(\ell^{12}\right)=0$ si et seulement si l'une des conditions suivantes est satisfaite :

$$
t_{\mathfrak{q}_{1}}= \pm t_{\mathfrak{q}_{2}} ; \quad t_{\mathfrak{q}_{1}}^{2}+t_{\mathfrak{q}_{2}}^{2} \pm t_{\mathfrak{q}_{1}} t_{\mathfrak{q}_{2}}=3 \ell ; \quad t_{\mathfrak{q}_{1}}^{2}+t_{\mathfrak{q}_{2}}^{2}=4 \ell
$$

Démonstration. La preuve de cette proposition repose sur la proposition2.1 ainsi que sur les relations de récurrence entre polynômes de Tchebychev. Elle n'est pas difficile. On ne traite que le cas où $\ell$ est inerte, les autres étant analogues. Supposons donc $\ell$ inerte dans $K$ avec $\ell \mathcal{O}_{K}=\mathfrak{q}$ et posons

$$
P_{\mathfrak{q}}(X)=X^{2}-t_{\mathfrak{q}} X+\ell^{2}=(X-\alpha)(X-\beta) .
$$

D'après la prop. 2.1, on a $|\alpha|=|\beta|=\ell$. Posons donc $\alpha=\ell e^{i \theta}$ avec $\theta \in \mathbf{R}$. D'après le lemme 3.1 , on a

$$
P_{\ell}^{*}(X)=\left(X-\alpha^{12}\right)\left(X-\beta^{12}\right) .
$$

D'où

$$
P_{\ell}^{*}(X)=X^{2}-\left(\alpha^{12}+\beta^{12}\right) X+\ell^{24}=X^{2}-2 \ell^{12} \cos (12 \theta) X+\ell^{24} .
$$

Or, $\cos (12 \theta)=T_{12}(\cos \theta)$ et $2 \ell \cos \theta=t_{\mathfrak{q}}$, d'où

$$
P_{\ell}^{*}(X)=X^{2}-2 \ell^{12} T_{12}\left(\frac{t_{\mathfrak{q}}}{2 \ell}\right) X+\ell^{24} .
$$

On en déduit immédiatement

$$
P_{\ell}^{*}\left(\ell^{12}\right)=2 \ell^{24}\left(1-T_{12}\left(\frac{t_{\mathfrak{q}}}{2 \ell}\right)\right) .
$$

Or, on a $1-T_{12}=8\left(1-T_{3}\right)\left(1+T_{3}\right) T_{3}^{2}$. D'où la factorisation

$$
P_{\ell}^{*}\left(\ell^{12}\right)=-\ell^{12} t_{\mathfrak{q}}^{2}\left(t_{\mathfrak{q}}^{2}-\ell^{2}\right)^{2}\left(t_{\mathfrak{q}}^{2}-4 \ell^{2}\right)\left(t_{\mathfrak{q}}^{2}-3 \ell^{2}\right)^{2}
$$

car

$$
T_{3}(X)=4 X^{3}-3 X ; \quad 1-T_{3}(X)=-(X-1)(2 X+1)^{2}
$$

et

$$
1+T_{3}(X)=(X+1)(2 X-1)^{2} .
$$

On en déduit que l'on a $P_{\ell}^{*}\left(\ell^{12}\right)=0$ si et seulement si $t_{\mathfrak{q}}=0, \pm \ell$ ou $\pm 2 \ell$. Autrement dit, si et seulement si $t_{\mathfrak{q}} \equiv 0(\bmod \ell)$ car $\left|t_{\mathfrak{q}}\right| \leq 2 \ell$. 


\subsection{Exemples}

On illustre dans les exemples suivants chacun des résultats du \$1.

Exemple 5.1 On suppose $K=\mathbf{Q}(\sqrt{5})$. On considère la courbe $E$ d'équation

$$
y^{2}=x^{3}+2 x^{2}+\omega x \quad \text { ò̀ } \quad \omega=\frac{1+\sqrt{5}}{2} .
$$

Alors, $\operatorname{Exc}(E / K)=\{2\}$.

Démonstration. On a

$$
\left\{\begin{array}{l}
c_{4}=2^{4}(4-3 \omega) \\
c_{6}=2^{6}(-8+9 \omega) \\
\Delta=-2^{6} \omega
\end{array}\right.
$$

Or, $\omega$ est une unité de $\mathcal{O}_{K}$. En particulier, la courbe $E$ a bonne réduction en dehors de (l'idéal premier) $2 \mathcal{O}_{K}$. On a :

$$
\left(v_{2}\left(c_{4}\right), v_{2}\left(c_{6}\right), v_{2}(\Delta)\right)=(4,6,6) .
$$

Donc $E$ a mauvaise réduction additive en 2. On note $\Phi_{2}$ son défaut de semistabilité en 2 . On a $v_{2}(j)=6$ et $3 v_{2}\left(c_{4}\right)=2 v_{2}\left(c_{6}\right)$. L'extension $K / \mathbf{Q}$ étant non ramifié en 2 , on a d'après [Cal04], $\left|\Phi_{2}\right|=4$ ou 8. Or, avec les notations de loc. cit. la condition (C2) n'est pas satisfaite. On en déduit que l'on a $\left|\Phi_{2}\right|=8$. Et, d'après le cor. 1.3. $\rho_{p}$ est irréductible pour tout nombre premier $p \geq 5$. La courbe $E$ a bonne réduction en l'idéal premier $7 \mathcal{O}_{K}$ et d'après le programme Trace0fFrobenius, on a $t_{7}=-12$. D'où

$$
P_{7}(X)=X^{2}-t_{7} X+49 \equiv X^{2}+1 \quad(\bmod 3) .
$$

Donc $\rho_{3}$ est également irréductible. La représentation $\rho_{2}$, en revanche, est réductible car $(0,0)$ est un point d'ordre 2 .

Exemple 5.2 On suppose $K=\mathbf{Q}(\sqrt{13})$. On considère la courbe $E$ d'équation

$$
y^{2}=x^{3}-(313+240 \omega) x-17 \quad \text { ò̀ } \quad \omega=\frac{1+\sqrt{13}}{2} .
$$

Alors, l'ensemble $\operatorname{Exc}(E / K)$ est vide.

Démonstration. On a

$$
\left\{\begin{array}{l}
c_{4}=2^{4} \cdot 3(11+8 \omega)^{2} \\
c_{6}=2^{5} \cdot 3^{3} \cdot 17 \\
\Delta=2^{4} \cdot 5(11+8 \omega)^{2}(213629+167568 \omega) .
\end{array}\right.
$$

De plus, $N_{K / \mathbf{Q}}(213629+167568 \omega)=-1153 \cdot 2430503$ et ni 1153 , ni 2430503 ne divisent $c_{4}$. Donc la courbe $E$ a mauvaise réduction multiplicative en un idéal premier au-dessus de 1153 et un idéal premier au-dessus de 2430503. Le nombre premier 2 est inerte dans $K$ et

$$
\left(v_{2}\left(c_{4}\right), v_{2}\left(c_{6}\right), v_{2}(\Delta)\right)=(4,5,4) .
$$


Donc $v_{2}(j)=8$ et d'après [Cal04, on a $\left|\Phi_{2}\right|=3,6$ ou 24. Comme par ailleurs,

$$
j^{\prime}=\frac{j}{2^{8}} \equiv-1 \quad(\bmod 4)
$$

la condition (C3) de loc. cit. est satisfaite avec $\gamma=1$ et $\left|\Phi_{2}\right|=3$ ou 6 (en fait $\left|\Phi_{2}\right|=6$ d'après loc. cit.). Puisque $f_{2}=2$ est pair, le cor. 1.3 ne s'applique pas. Cependant, en l'idéal premier $\mathfrak{q}_{17}=(15+4 \sqrt{13}) \mathcal{O}_{K}$, on a

$$
\left(v_{\mathfrak{q}_{17}}\left(c_{4}\right), v_{\mathfrak{q}_{17}}\left(c_{6}\right), v_{\mathfrak{q}_{17}}(\Delta)\right)=(2,1,2) .
$$

Donc $E$ a mauvaise réduction additive en $\mathfrak{q}_{17}$ avec potentiellement bonne réduction. Son défaut de semi-stabilité $\Phi_{\mathfrak{q}_{17}}$ est d'ordre 6 ([Ser72, p.312]). Or, 6 ne divise pas $N\left(\mathfrak{q}_{17}\right)-1=16$. Donc, d'après la prop. 1.2, la représentation $\rho_{p}$ est irréductible pour tout nombre premier $p \geq 3$ et $p \neq 1$. Si $\mathfrak{q}_{3}$ désigne un idéal divisant 3 , alors $E$ a bonne réduction en $\mathfrak{q}_{3}$ et d'après le programme TraceOfFrobenius, on a

$$
t_{\mathfrak{q}_{3}}=-3 .
$$

Donc le polynôme $P_{\mathfrak{q}_{3}}(X)=X^{2}+3 X+3$ est irréductible modulo 2 et 17 . On en déduit le résultat.

Exemple 5.3 On suppose $K=\mathbf{Q}(\sqrt{-1})$. Pour tout entier $a \in \mathbf{Z}[\sqrt{-1}]$, on considère la courbe $E_{a}$ d'équation

$$
y^{2}=x^{3}+a x+a .
$$

Supposons que l'on ait $v_{\mathfrak{q}_{2}}(a)=0,1,3,4,5,6$ ou 7 , où $\mathfrak{q}_{2}$ est l'unique idéal de $\mathcal{O}_{K}$ au-dessus de 2. Alors, l'ensemble $\operatorname{Exc}\left(E_{a} / K\right)$ est contenu dans $\{2,3\}$.

Démonstration. On a

$$
\left\{\begin{array}{l}
c_{4}\left(E_{a}\right)=-2^{4} \cdot 3 \cdot a \\
c_{6}\left(E_{a}\right)=-2^{5} \cdot 3 \cdot a \\
\Delta\left(E_{a}\right)=-2^{4} \cdot a^{2}(4 a+27) .
\end{array}\right.
$$

En particulier, $v_{\mathfrak{q}_{2}}\left(c_{4}\left(E_{a}\right)\right)=8+v_{\mathfrak{q}_{2}}(a), v_{\mathfrak{q}_{2}}\left(\Delta\left(E_{a}\right)\right)=8+2 v_{\mathfrak{q}_{2}}(a)$ et

$$
v_{\mathfrak{q}_{2}}\left(j\left(E_{a}\right)\right)=16+v_{\mathfrak{q}_{2}}(a) \geq 0 .
$$

Donc $E_{a}$ a mauvaise réduction additive en $\mathfrak{q}_{2}$ avec potentiellement bonne réduction. Par ailleurs, 2 est ramifié dans $K$ (donc $f_{\mathfrak{q}_{2}}=1$ est impair) et

$$
v_{\mathfrak{q}_{2}}\left(j\left(E_{a}\right)\right) \in\{16,17,19,20,21,22,23\} .
$$

Donc, d'après Bil09, on a $\left|\Phi_{\mathfrak{q}_{2}}\right| \in\{3,6,8,24\}$. On conclut avec le cor. 1.3.

Avec les notations de l'exemple précédent, lorsque $v_{\mathfrak{q}_{2}}(a)=2$ ou $\geq 8$, le corollaire 1.3 ne s'applique pas toujours. On traite ci-dessous un exemple dans le cas où $v_{\mathfrak{q}_{2}}(a)=2$.

Exemple 5.4 On suppose $K=\mathbf{Q}(\sqrt{-1})$. On considère la courbe $E$ d'équation

$$
y^{2}=x^{3}+2(3+2 \sqrt{-1}) x+2(3+2 \sqrt{-1}) .
$$

Alors, l'ensemble $\operatorname{Exc}(E / K)$ est vide. 
Démonstration. On a

$$
\left\{\begin{array}{l}
c_{4}=-2^{5} \cdot 3(3+2 \sqrt{-1}) \\
c_{6}=-2^{6} \cdot 3^{3}(3+2 \sqrt{-1}) \\
\Delta=-2^{6}(3+2 \sqrt{-1})^{2} \cdot(51+16 \sqrt{-1}) .
\end{array}\right.
$$

On a $2 \mathcal{O}_{K}=\mathfrak{q}_{2}^{2}$ où $\mathfrak{q}_{2}=(1+\sqrt{-1}) \mathcal{O}_{K}$ et

$$
\left(v_{\mathfrak{q}_{2}}\left(c_{4}\right), v_{\mathfrak{q}_{2}}\left(c_{6}\right), v_{\mathfrak{q}_{2}}(\Delta)\right)=(10,12,12) .
$$

Donc, d'après Pap93, l'équation (18) correspond à un cas 6 ou 7 de Tate. En particulier, il est minimal en $\mathfrak{q}_{2}$ et $E$ a réduction additive en $\mathfrak{q}_{2}$. Déterminons à présent l'ordre $\left|\Phi_{\mathfrak{q}_{2}}\right|$ de son défaut de semi-stabilité en $\mathfrak{q}_{2}$. On a $v_{\mathfrak{q}_{2}}(j)=18$ et $\pi=1+\sqrt{-1}$ est une uniformisante de $K_{\mathfrak{q}_{2}}$. De plus,

$$
\frac{c_{4}}{\pi^{10}} \equiv 1+\pi \quad(\bmod 2)
$$

Donc, d'après [Bil09, th. 2], on a $\left|\Phi_{\mathfrak{q}_{2}}\right|=4$. On ne peut donc pas appliquer le cor. 1.3 .

Notons $\mathfrak{q}_{13}$ l'idéal premier de $\mathcal{O}_{K}$ engendré par $3+2 \sqrt{-1}$. On a

$$
\left(v_{\mathfrak{q}_{13}}\left(c_{4}\right), v_{\mathfrak{q}_{13}}(\Delta)\right)=(1,2),
$$

d'où $v_{\mathfrak{q}_{13}}(j)=1$. L'équation (18) est minimale en $\mathfrak{q}_{13}$ et $E$ a mauvaise réduction additive en $\mathfrak{q}_{13}$ avec potentiellement bonne réduction. Son défaut de semistabilité est d'ordre 6 (c.f. [Ser72, p.312]). Comme 6 divise $N\left(\mathfrak{q}_{13}\right)-1=12$, la prop. 1.2 ne donne aucune majoration de $\operatorname{Exc}(E / K)$. Par ailleurs, on a $v_{\mathfrak{q}_{13}}(\Delta)=2$ et d'après Kra97, lem.1], $E$ n'a pas potentiellement bonne réduction de hauteur 2 en $\mathfrak{q}_{13}$.

Notons $\mathfrak{q}_{2857}$ l'idéal premier de $\mathcal{O}_{K}$ engendré par $51+16 \sqrt{-1}$. La courbe $E$ a mauvaise réduction multiplicative en $\mathfrak{q}_{2857}$. En dehors de $\mathfrak{q}_{2}, \mathfrak{q}_{13}$ et $\mathfrak{q}_{2857}$, la courbe $E$ a bonne réduction.

Vu l'étude précédente, aucun des résultats « uniformes »du \$1 ne s'applique. Pour cette courbe, on a donc recours au critère du th. 1. Soit $p \geq 5$ un nombre premier exceptionnel. D'après le programme Trace0fFrobenius, on a

$$
\left\{t_{\mathfrak{q}}\right\}_{\mathfrak{q} \mid 5}=\{-2,1\} \quad \text { et } \quad t_{7}=6 .
$$

D'après le th. 1 appliquée à $\ell=5$ et $\ell=7, p$ divise chacun des entiers $B_{5}$ et $B_{7}$. Or, d'après le programme ExceptionalPrimes, on a

$$
B_{5}=2^{28} \cdot 3^{16} \cdot 5^{39} \cdot 11^{2} \cdot 17 \cdot 61 \cdot 73 \cdot 277 \cdot 397 \cdot 557 \cdot 653 \cdot 757 \cdot 23833
$$

et

$$
B_{7}=2^{14} \cdot 3^{8} \cdot 5^{2} \cdot 7^{13} \cdot 11 \cdot 13^{5} \cdot 37^{2} \cdot 2089 \cdot 2689 \cdot 3889 .
$$

D'où

$$
p \in \mathfrak{S}_{5}^{(2)} \cap \mathfrak{S}_{7}^{(2)}=\{2,3,5,11\} .
$$

Il ne reste donc plus qu'à traiter les cas $p=2,3,5$ et 11 . Or, $E$ a bonne réduction en l'idéal premier $3 \mathcal{O}_{K}$ et d'après le programme TraceOfFrobenius, on a

$$
P_{3}(X)=X^{2}+3 X+9 .
$$


Donc $P_{3}$ est irréductible modulo 2,5 et 11 . Et, si $\mathfrak{q}_{5}$ est un idéal premier audessus de 5 , on a $t_{\mathfrak{q}_{5}}=-2$ ou 1 , et

$$
P_{\mathfrak{q}_{5}}(X) \equiv X^{2}+2 X+2 \quad(\bmod 3) .
$$

Donc $P_{\mathfrak{q}_{5}}$ est irréductible modulo 3 . On en déduit que $\rho_{p}$ est également irréductible pour $p=2,3,5$ et 11 . D'où le résultat.

Exemple 5.5 On suppose $K=\mathbf{Q}(\sqrt{2})$ et on pose

$$
\left\{\begin{array}{l}
A=-3^{3} \cdot 5 \cdot 17^{3}(428525+303032 \sqrt{2}) \\
B=2 \cdot 3^{3} \cdot 5 \cdot 17^{3}(62176502533+43965551956 \sqrt{2}) .
\end{array}\right.
$$

On considère la courbe E d'équation

$$
y^{2}=x^{3}+A x+B .
$$

Alors, $\operatorname{Exc}(E / K)=\{13\}$.

Démonstration. On vérifie que pour le modèle choisi, on a

$$
N_{K / \mathbf{Q}}(\Delta)=-2^{25} \cdot 3^{18} \cdot 5^{4} \cdot 7^{2} \cdot 17^{15} \cdot 23^{6} \cdot 79^{6} .
$$

En particulier, la courbe $E$ a bonne réduction en les idéaux premiers divisant $11,13,19,29$ et 41 et d'après le programme TraceOfFrobenius, on a

$$
t_{11}=4 ; \quad t_{13}=-14 \quad t_{19}=26 ; \quad t_{29}=1 \quad \text { et } \quad\left\{t_{\mathfrak{q}}\right\}_{\mathfrak{q} \mid 41}=\{-3,2\} .
$$

Soit $p$ un nombre premier exceptionnel n'appartenant pas à l'ensemble

$$
\{2,3,5,7,17,23,79\} \text {. }
$$

Alors, d'après le corollaire 1.1, on a en particulier

$$
p \in \mathfrak{S}_{11}^{(2)} \cap \mathfrak{S}_{13}^{(2)} .
$$

D'où, d'après le programme ExceptionalPrimes,

$$
p \in\{2,3,5,7,13\} .
$$

Autrement dit, il ne reste plus qu'à traiter les cas où $p=2,3,5,7,13,17,23$ et 79. Or le polynôme $P_{11}$ est irréductible modulo 5,23 et 79 . De même, $P_{13}$ est irréductible modulo $7, P_{19}$ modulo 17 et $P_{29}$ modulo 2 . Si $\mathfrak{q}_{41}$ désigne l'idéal premier de $\mathcal{O}_{K}$ au-dessus de 41 tel que $t_{\mathfrak{q}_{41}}=2$, alors $P_{\mathfrak{q}_{41}}$ est irréductible modulo 3 . On en déduit que 2, 3, 5, 7, 17, 23 et 79 ne sont pas exceptionnels. En revanche 13 est un nombre premier exceptionnel. En effet, la courbe modulaire $X_{0}(13)$ paramétrisant les courbes elliptiques munies d'un sous-groupe stable d'ordre 13 est de genre 0 et un isomorphisme avec $\mathbf{P}^{1}$ est donné par la fraction rationnelle suivante ([Mes80, §2.2]) :

$$
j\left(X_{0}(13)\right)(X)=\frac{\left(X^{2}+5 X+13\right)\left(X^{4}+7 X^{3}+20 X^{2}+19 X+1\right)^{3}}{X} .
$$

On vérifie alors que l'on a $j\left(X_{0}(13)\right)(\sqrt{2})=j$. Cela montre que 13 est exceptionnel et le résultat annoncé. 
Exemple 5.6 On suppose $K=\mathbf{Q}(\sqrt{3})$ et on pose

$$
\left\{\begin{array}{l}
a_{1}=2^{2} \cdot 7 \sqrt{3}(1+2 \sqrt{3})(2-\sqrt{3})=252-112 \sqrt{3} \\
a_{4}=2 \cdot 3^{2} \cdot 7^{2} \sqrt{3}(1+2 \sqrt{3})^{2}=10584+11466 \sqrt{3} \\
a_{6}=2^{3} \cdot 3 \cdot 7^{4} \sqrt{3}(1+2 \sqrt{3})^{4}(7-4 \sqrt{3})=-24202080+15616104 \sqrt{3}
\end{array}\right.
$$

On considère la courbe E d'équation

$$
y^{2}+a_{1} x y=x^{3}+a_{4} x+a_{6} .
$$

Alors, la courbe $E$ a des multiplications complexes par le corps $\mathbf{Q}(\sqrt{-1})$ et $\operatorname{Exc}(E / K)=\{2,3\}$.

Démonstration. On a

$$
\left\{\begin{array}{l}
c_{4}=-2^{5} \cdot 3^{2} \cdot 7^{2} \cdot 11^{2}(-1+2 \sqrt{3})(2-3 \sqrt{3})^{3} \varepsilon^{-2} \\
c_{6}=2^{9} \cdot 3^{3} \cdot 7^{4} \cdot 11^{3}(1+2 \sqrt{3})(2-3 \sqrt{3})^{3} \varepsilon^{-4} \\
\Delta=-2^{9} \cdot 3^{4} \cdot 7^{6} \cdot 11^{6} \sqrt{3}(2-3 \sqrt{3})^{6} \varepsilon^{-4}
\end{array}\right.
$$

où $\varepsilon=2+\sqrt{3}$ est l'unité fondamentale de $\mathbf{Q}(\sqrt{3})$. On en déduit que

$$
\begin{aligned}
j & =2^{6} \cdot 3 \cdot \sqrt{3}(-1+2 \sqrt{3})^{3}(2-3 \sqrt{3})^{3} \varepsilon^{-2} \\
& =76771008-44330496 \sqrt{3}
\end{aligned}
$$

est entier de polynôme minimal sur $\mathbf{Q}$

$$
P(X)=X^{2}-153542016 X-1790957481984 .
$$

Vérifions que $E$ a des multiplications complexes par l'ordre de $\mathbf{Q}(\sqrt{-1})$ de conduteur 3. En effet, il n'y a qu'un nombre fini de classes d'isomorphisme de courbes elliptiques ayant des multiplications complexes par un ordre de discriminant $D$ fixé. De plus, le polynôme minimal sur $\mathbf{Q}$ de l'invariant modulaire d'une telle courbe elliptique est

$$
\Phi_{D}(X)=\prod_{a, b, c}\left(X-j\left(\frac{-b+\sqrt{D}}{2 a}\right)\right),
$$

où $j$ désigne la fonction modulaire et $(a, b, c)$ parcourt l'ensemble des triplets d'entiers tels que la forme quadratique $a x^{2}+b x y+c y^{2}$ soit primitive positive réduite de discriminant $D$ ([Coh93, Th. 7.2.14]). Dans le cas où $D=-36$, on a exactement deux représentants des classes d'équivalence de telles formes quadratiques donnés par

$$
x^{2}+9 y^{2} \text { et } 2 x^{2}+2 x y+5 y^{2} .
$$

On vérifie alors que l'on a

$$
\begin{aligned}
\Phi_{-36}(X) & =(X-j(3 \sqrt{-1}))\left(X-j\left(\frac{-1+3 \sqrt{-1}}{2}\right)\right) \\
& =X^{2}-153542016 X-1790957481984=P(X)
\end{aligned}
$$


et

$$
j=j\left(\frac{-1+3 \sqrt{-1}}{2}\right) .
$$

Cela établit l'assertion.

Par ailleurs, d'après l'expression des coefficients $c_{4}, c_{6}$ et $\Delta$ ci-dessus, $E$ a réduction additive en l'idéal $\mathfrak{q}_{3}=\sqrt{3} \mathcal{O}_{K}$ et

$$
\left(v_{\mathfrak{q}_{3}}\left(c_{4}\right), v_{\mathfrak{q}_{3}}\left(c_{6}\right), v_{\mathfrak{q}_{3}}(\Delta)\right)=(4,6,9) .
$$

En particulier, on a d'après [Kra90, th. 1], $\left|\Phi_{\mathfrak{q}_{3}}\right|=4$ ou 12. Donc, d'après le cor. 1.4, la représentation $\rho_{p}$ est irréductible pour tout nombre premier $p \geq 5$. Par ailleurs, la courbe modulaire $X_{0}(3)$ paramétrisant les courbes elliptiques munies d'un sous-groupe stable d'ordre 3 est de genre 0 et un isomorphisme avec $\mathbf{P}^{1}$ est donné par la fraction rationnelle suivante :

$$
j\left(X_{0}(3)\right)(X)=\frac{(X+3)^{3}(X+27)}{X} .
$$

On vérifie alors que l'on a $j\left(X_{0}(3)\right)(243-162 \sqrt{3})=j$. Cela montre que 3 est exceptionnel. On vérifie enfin que le point de coordonnées affines

$$
\left\{\begin{array}{l}
x=-2^{2} \cdot 7(15+8 \sqrt{3}) \\
y=2^{3} \cdot 3 \cdot 7^{2}(13+4 \sqrt{3})
\end{array}\right.
$$

est un point d'ordre 2 de $E$. En particulier, 2 est exceptionnel. D'où le résultat.

Remarque. Pour montrer la finitude de l'ensemble exceptionnel, on aurait pu appliquer directement le cor.1.1(au lieu du cor.1.4) par exemple avec le nombre premier $\ell=5$ pour lequel $B_{5} \neq 0$.

Dans les deux derniers exemples suivants, on a utilisé magma ([BCP97]) pour calculer certains coefficients de la fonction $L$ de $E$.

Exemple 5.7 On considère $K=\mathbf{Q}\left(\cos \left(\frac{2 \pi}{9}\right)\right)$ le corps cubique cyclique de conducteur 9 et la courbe $E$ d'équation

$$
y^{2}=x^{3}+2(1+\alpha)^{2} x+24 \alpha(2+\alpha),
$$

où $\alpha=2 \cos \left(\frac{2 \pi}{9}\right)$ est racine du polynôme $X^{3}-3 X+1$. Alors, l'ensemble $\operatorname{Exc}(E / K)$ est vide.

Démonstration. On a $D_{K}=3^{4}$ et $3 \mathcal{O}_{K}=\mathfrak{q}_{3}^{3}$ où $\mathfrak{q}_{3}$ est l'idéal de $\mathcal{O}_{K}$ engendré par $1+\alpha$. De plus, on vérifie que l'on a

$$
\left\{\begin{array}{l}
c_{4}=-2^{5}(1+\alpha)^{5}\left(1+\alpha-\alpha^{2}\right) \\
c_{6}=-2^{8}(1+\alpha)^{12}\left(1+\alpha-\alpha^{2}\right)^{3} \\
\Delta=-2^{9}(1+\alpha)^{6} \cdot 5 \cdot 11
\end{array}\right.
$$

et $1+\alpha-\alpha^{2}$ est une unité de $\mathcal{O}_{K}$. La courbe $E$ a mauvaise réduction multiplicative en les idéaux premiers $5 \mathcal{O}_{K}$ et $11 \mathcal{O}_{K}$. En l'idéal premier $\mathfrak{q}_{3}$, la courbe a mauvaise réduction additive avec potentiellement bonne réduction et $v_{\mathfrak{q}_{3}}\left(\Delta_{\mathfrak{q}_{3}}\right)=$ $v_{\mathfrak{q}_{3}}(\Delta)=6$. Donc, d'après [Kra90, th.1], on a $\left|\Phi_{\mathfrak{q}_{3}}\right|=2$ ou 6. En l'idéal premier $2 \mathcal{O}_{K}$, la courbe $E$ a mauvaise réduction additive avec potentiellement bonne réduction et

$$
\left(v_{2}\left(c_{4}\right), v_{2}\left(c_{6}\right), v_{2}(\Delta)\right)=(5,8,9)
$$


d'où $v_{2}(j)=6$ et $2 v_{2}\left(c_{6}\right)=3 v_{2}\left(c_{4}\right)+1$. Comme de plus

$$
j^{\prime}=\frac{j}{2^{6}}=\frac{(1+\alpha)^{9}\left(1+\alpha-\alpha^{2}\right)^{3}}{5 \cdot 11}=\frac{3^{3}}{5 \cdot 11} \equiv 1 \quad(\bmod 4),
$$

d'après Cal04, on a $\left|\Phi_{2}\right|=4$. Partout ailleurs, la courbe $E$ a bonne réduction.

Vu l'étude précédente, aucun des résultats « uniformes »du \$1 ne s'applique. Les nombres premiers $17,19,37$ et 53 sont (totalement) décomposés dans $K$ et on vérifie que l'on a

$$
\begin{array}{ll}
\left\{t_{\mathfrak{q}}\right\}_{\mathfrak{q} \mid 17}=\{-3,-3,3\} ; & \left\{t_{\mathfrak{q}}\right\}_{\mathfrak{q} \mid 19}=\{-5,-5,5\} \\
\left\{t_{\mathfrak{q}}\right\}_{\mathfrak{q} \mid 37}=\{-7,-7,7\} ; & \left\{t_{\mathfrak{q}}\right\}_{\mathfrak{q} \mid 53}=\{-3,3,3\} .
\end{array}
$$

Soit $p$ un nombre premier exceptionnel $\geq 5$. D'après le th. 1, on a en particulier,

$$
p \in \mathfrak{S}_{17}^{(3)} \cap \mathfrak{S}_{19}^{(3)} \cap \mathfrak{S}_{37}^{(3)}=\{2,3,5\} .
$$

Il ne reste donc plus qu'à traiter les cas où $p=2,3$ ou 5 . Or, si $\mathfrak{q}_{53}$ désigne un idéal premier de $\mathcal{O}_{K}$ au-dessus de 53 , le polynôme $P_{\mathfrak{q}_{53}}$ est irréductible modulo 2 et 5 . Par ailleurs, l'idéal $7 \mathcal{O}_{K}$ est premier et $t_{7}=-36$, donc le polynôme

$$
P_{7}(X)=X^{2}+36 X+7^{3}
$$

est irréductible modulo 3 . On en déduit le résultat annoncé.

Exemple 5.8 On considère $K=\mathbf{Q}(\sqrt{-3}, \sqrt{-7})$ et E la courbe d'équation

$$
y^{2}=x^{3}+a_{4} x+a_{6}
$$

ò̀

$$
\left\{\begin{array}{l}
a_{4}=\frac{81}{4} \cdot(69+43 \sqrt{-3}+29 \sqrt{-7}+17 \sqrt{21}) \\
a_{6}=162 \cdot(207-84 \sqrt{-3}-54 \sqrt{-7}+46 \sqrt{21}) .
\end{array}\right.
$$

Alors, $\operatorname{Exc}(E / K)=\{2,3,5\}$.

Démonstration. La courbe $E$ a la propriété particulière d'être une Q-courbe, c'est-à-dire, d'être isogène à ses conjuguées galoisiennes. Plus précisément, c'est une Q-courbe (sans multiplication complexe) de conducteur $2 \mathcal{O}_{K}$ dont tous les endomorphismes sont définis sur $K$ (cf. GG10, ex. 13] et [GL01]). En les idéaux au-dessus de 2, elle a mauvaise réduction multiplicative. En particulier, aucun des résultats 《uniformes » du @1 ne s'applique. Montrons à présent que le critère $\mathrm{du}$ th. 1 est lui aussi insuffisant pour traiter cette courbe. On doit montrer que pour tout nombre premier $\ell \geq 3$, l'entier $B_{\ell}$ est nul, autrement dit, que $\ell^{24}$ est racine de $P_{\ell}^{*}$ (lemme 3.4). D'après les propriétés de $E$ rappelées ci-dessus, on a $P_{\mathfrak{q}}=P_{\mathfrak{q}^{\prime}}$ pour tout couple $\left(\mathfrak{q}, \mathfrak{q}^{\prime}\right)$ d'idéaux premiers divisant $\ell$. Or $D_{K}=3^{2} \cdot 7^{2}$, donc si $\ell \neq 3,7, \ell \mathcal{O}_{K}$ se décompose en un produit de 2 ou 4 idéaux premiers. On a alors respectivement

$$
P_{\ell}^{*}=\left(P_{\mathfrak{q}}^{(12)}\right)^{* 2} \quad \text { et } \quad P_{\ell}^{*}=\left(P_{\mathfrak{q}}^{(12)}\right)^{* 4} .
$$


Or, dans le premier cas, les racines complexes $\alpha$ et $\beta$ de $P_{\mathfrak{q}}$ satisfont $\alpha \beta=\ell^{2}$ et dans le second, $\alpha \beta=\ell$. On en déduit le résultat voulu dans ce cas. Par ailleurs, on vérifie que l'on a $P_{3}^{*}(X)=\left(X-3^{24}\right)^{2}$ et

$$
P_{7}^{*}(X)=\left(X-7^{24}\right)^{2} \cdot\left(X^{2}-371728108602950083202 X+7^{48}\right),
$$

d'où la nullité de $B_{\ell}$ pour tout $\ell$ de bonne réduction. Pour cette courbe, on a donc recours au critère du th. 2. Le nombre de classes $h$ de $K$ est 1 . On considère l'idéal premier $\mathfrak{q}_{5}$ au-dessus de 5 engendré par une racine $\gamma_{\mathfrak{q}_{5}}$ du polynôme $\mathfrak{m}_{\gamma_{\mathfrak{q}_{5}}}(X)=X^{4}+17 X^{2}+25$. On a alors,

$$
P_{\mathfrak{q}_{5}}(X)=X^{2}+4 X+25 \quad \text { d'où } \quad P_{\mathfrak{q}_{5}}^{(12)}(X)=X^{2}-2 \cdot 47 \cdot 1163039 X+5^{24}
$$

et

$$
\mathfrak{m}_{\gamma_{\mathfrak{q}_{5}}}^{(12)}(X)=\left(X^{2}-2 \cdot 73 \cdot 19441 X+5^{12}\right)^{2}
$$

puis,

$$
\left(\mathfrak{m}_{\gamma_{q_{5}}}^{(12)}\right)^{* 2}(X)=\left(X-5^{12}\right)^{8} \cdot\left(X^{2}-2 \cdot 79 \cdot 127 \cdot 337 \cdot 1191313 X+5^{24}\right)^{4} .
$$

On en déduit que l'on a

$$
\begin{array}{r}
R_{\mathfrak{q}_{5}}=2^{126} \cdot 3^{100} \cdot 5^{225} \cdot 7^{21} \cdot 11 \cdot 13^{8} \cdot 19 \cdot 37^{11} \cdot 41^{8} \cdot 59^{16} \cdot 103 \cdot 109^{8} \cdot 149^{8} \\
\cdot 193 \cdot 373^{2} \cdot 2137 \cdot 4201^{2} \cdot 7753^{2} \cdot 24061^{2}
\end{array}
$$

On recommence ensuite ces mêmes calculs avec l'idéal premier $\mathfrak{q}_{7}$ au-dessus de 7 engendré par une racine $\gamma_{\mathfrak{q}_{7}}$ du polynôme $\mathfrak{m}_{\gamma_{\mathfrak{q}_{7}}}(X)=X^{4}+4 X^{3}+11 X^{2}+14 X+7$. On a alors $P_{\mathfrak{q}_{7}}(X)=X^{2}+2 X+7$ et

$$
\begin{array}{r}
R_{\mathfrak{q}_{7}}=2^{105} \cdot 3^{59} \cdot 5^{26} \cdot 7^{116} \cdot 11^{2} \cdot 13^{2} \cdot 17^{8} \cdot 23^{8} \cdot 31 \cdot 79 \cdot 137^{2} \cdot 191^{4} \cdot 193 \\
\cdot 463 \cdot 487^{2} \cdot 673 \cdot 1033^{2} \cdot 1471 \cdot 2953 \cdot 3697 .
\end{array}
$$

Après ces deux itérations du th. 2, on a donc montré l'inclusion

$$
\operatorname{Exc}(E / K) \subset\{2,3,5,7,11,13,193\} .
$$

Notons respectivement, $\mathfrak{q}_{3}$ et $\mathfrak{q}_{17}$ un idéal premier au-dessus de 3 et de 17. Alors, le polynôme $P_{\mathfrak{q}_{3}}(X)=X^{2}+9$ est irréductible modulo 7 et 11 et le polynôme $P_{\mathfrak{q}_{17}}(X)=X^{2}+10 X+289$ est irréductible modulo 193. De même, le polynôme $P_{\mathfrak{q}_{5}}$ ci-dessus est irréductible modulo 13. Enfin, 2, 3 et 5 sont exceptionnels car ce sont les degrés des isogénies de $E$ vers ses trois conjuguées galoisiennes (loc. cit.).

D'où le résultat.

\section{Références}

[Bil09] N. Billerey. Semi-stabilité des courbes elliptiques. Dissertationes Math., 468 :57, 2009.

[BP08] Y. Bilu et P. Parent. Serre's uniformity problem in the split cartan case. ArXiv e-prints, 2008. 
[BCP97] W. Bosma, J. Cannon et C. Playoust. The Magma algebra system. I. The user language. J. Symbolic Comput., 24(3-4) :235-265, 1997. Computational algebra and number theory (London, 1993).

[Bou81] N. Bourbaki. Éléments de mathématique, volume 864 of Lecture Notes in Mathematics. Masson, Paris, 1981. Algèbre. Chapitres 4 à 7.

[Cal04] É. Cali. Défaut de semi-stabilité des courbes elliptiques dans le cas non ramifié. Canad. J. Math., 56(4) :673-698, 2004.

[Coh93] H. Cohen. A course in computational algebraic number theory, volume 138 of Graduate Texts in Mathematics. Springer-Verlag, Berlin, 1993.

[Coh00] _. Advanced topics in computational number theory, volume 193 of Graduate Texts in Mathematics. Springer-Verlag, New York, 2000.

[Dav08] A. David. Caractère d'isogénie et borne uniforme pour les homothéties. Thèse de l'université de Strasbourg, 2008.

[GL01] J. González et J. C. Lario. Q-curves and their Manin ideals. Amer. J. Math., 123(3) :475-503, 2001.

[GG10] E. Gonzalez-Jimenez et X. Guitart. On the modularity level of modular abelian varieties over number fields. ArXiv e-prints, 2010.

[Kra90] A. Kraus. Sur le défaut de semi-stabilité des courbes elliptiques à réduction additive. Manuscripta Math., 69(4) :353-385, 1990.

[Kra96] _ Courbes elliptiques semi-stables et corps quadratiques. $J$. Number Theory, 60 :245-253, 1996.

[Kra97] - Détermination du poids et du conducteur associés aux représentations des points de $p$-torsion d'une courbe elliptique. Dissertationes Math., 364, 1997.

[Kra07] - Courbes elliptiques semi-stables sur les corps de nombres. Int. J. Number Theory, 3(4) :611-633, 2007.

[KO92] A. Kraus et J. Oesterlé. Sur une question de B. Mazur. Math. Ann., $293: 259-275,1992$.

[Lan87] S. Lang. Elliptic functions, volume 112 of Graduate Texts in Mathematics. Springer-Verlag, New York, second edition, 1987. With an appendix by J. Tate.

[Maz78] B. Mazur. Rational Isogenies of Prime Degree. Invent. Math., 44, 1978.

[Mes80] J.-F. Mestre. Points rationnels de la courbe modulaire $X_{0}(169)$. Ann. Inst. Fourier (Grenoble), 30(2) :17-27, 1980.

[Mom95] F. Momose. Isogenies of prime degree over number fields. Compositio Math., 97(3) :329-348, 1995.

[Neu86] J. Neukirch. Class field theory, volume 280 of Grundlehren der Mathematischen Wissenschaften [Fundamental Principles of Mathematical Sciences]. Springer-Verlag, Berlin, 1986.

[Pap93] I. Papadopoulos. Sur la classification de Néron des courbes elliptiques en caractéristique résiduelle 2 et 3 . J. Number Theory, 44(2) :119-152, 1993.

[Pel01] F. Pellarin. Sur une majoration explicite pour un degré d'isogénie liant deux courbes elliptiques. Acta Arith., 100(3) :203-243, 2001. 
[Ser68] J.-P. Serre. Abelian l-adic representations and elliptic curves. McGill University. W. A. Benjamin, Inc., New York-Amsterdam, 1968.

[Ser72] _ Propriétés galoisiennes des points d'ordre fini des courbes elliptiques. Invent. Math., 15 :259-331, 1972.

[Ser79] _ Points rationnels des courbes modulaires $X_{0}(N)$ [d'après Barry Mazur]. In Séminaire Bourbaki, 30e année (1977/78), volume 710 of Lecture Notes in Math., pages Exp. No. 511, pp. 89-100. Springer, Berlin, 1979.

[ST68] J.-P. Serre et J. Tate. Good reduction of abelian varieties. Ann. of Math., 88 :492-517, 1968.

[Sil92] J. H. Silverman. The arithmetic of elliptic curves, volume 106 of Graduate Texts in Mathematics. Springer-Verlag, 1992.

[Tat75] J. Tate. Algorithm for determining the type of a singular fiber in an elliptic pencil. in Modular functions of one variable, Lect. Notes in Math., 273 :33-52, 1975. 